\title{
Technische Aspekte des automatisierten Fahrens am Projekt des autonomen Shuttlebusses in Bad Birnbach
}

\author{
Jan Christopher Kolb, Lothar Wech, Martin Schwabe, \\ Christopher Ruzok und Christoph Trost
}

Die Entwickler von selbstfahrenden Fahrzeugen sind sich einig, dass es nach heutigem Stand der Technik über eine Milliarde Testkilometer bedarf, um ein autonom fahrendes Fahrzeug zu entwickeln. Daher überrascht es nicht, dass der ,,autonome“ Shuttlebus in Bad Birnbach lediglich als teilautomatisiert einzustufen ist. Dennoch ist das Projekt wertvoll, um Erkenntnisse für die Entwicklung von autonomen Fahrzeugen zu erlangen und deren Einsatz im öffentlichen Personennahverkehr (ÖPNV) beurteilen zu können. Im vorliegenden Teilprojekt wurden innerhalb von vier Monaten über dreihundert Situationen dokumentiert, in denen die Fahrt unplanmäßig unterbrochen wurde. Diese wurden anschließend ausgewertet und teilweise in Versuchen nachgestellt. Es hat sich gezeigt, dass der Shuttlebus im aktuellen Betriebsmodell unfallfrei fahren kann. Allerdings ist für das unfall- und unterbrechungsfreie Fahren der durch die Zulassungsauflagen vorgeschriebene Operator an Bord zwingend erforderlich. Dieser muss an verschiedenen Stellen der Strecke - inklusive der Haltestellen - die Weiterfahrt bestätigen, den Bus um Hindernisse lenken und bei einem drohenden Unfall eingreifen. Ohne Operator käme es zur Kollision, da die Technik des Busses nicht alle kritischen Situationen erkennt. Der Bus kann im aktuellen Entwicklungsstand weder fahrerlos betrieben noch als zuverlässige Ergänzung im ÖPNV eingesetzt werden. Der Bus stellt dennoch einen wichtigen Schritt in Richtung autonomen Fahrens dar, welches weiterhin im Rahmen zukünftiger Forschungs- und Entwicklungsprojekte vorangetrieben werden muss.

J. C. Kolb $(\bowtie) \cdot$ M. Schwabe $\cdot$ C. Ruzok $\cdot$ C. Trost

Technische Hochschule Ingolstadt, Forschungszentrum CARISSMA, Ingolstadt, Deutschland E-Mail: janchristopher.kolb@carissma.eu; martin.schwabe@thi.de; christopher.ruzok@ carissma.eu; christoph.trost@ carissma.eu

L. Wech

Technische Hochschule Ingolstadt, Ingolstadt, Deutschland

E-Mail: lothar.wech@thi.de

A. Riener et al. (Hrsg.), Autonome Shuttlebusse im ÖPNV, https://doi.org/10.1007/978-3-662-59406-3_5 


\subsection{Einleitung}

Weltweit betreiben Fahrzeughersteller, Start-Ups und Großkonzerne aus der IT-Branche Entwicklungen mit dem Ziel des automatisierten Fahrens. Hinzu kommen Unternehmen, die die entwickelte Technik für ihre Zwecke nutzen und unter realen Bedingungen zum Einsatz bringen. Bisher war aber noch kein autonomer Shuttlebus auf deutschen öffentlichen Straßen darunter. Abgesehen von einzelnen Testfahrten der Entwickler gibt es zum Start des Projektes „Einführung und Betrieb eines selbstfahrenden Shuttleverkehrs im ÖPNV in Bad Birnbach“ in Deutschland auch keine anderen Projekte mit autonom fahrenden Fahrzeugen. Somit eignet sich das Projekt in Bad Birnbach unter anderem für Forschungen hinsichtlich des Betriebs, der Infrastruktur und der eingesetzten Technik, sowie deren Auswirkungen auf den Einsatz sogenannter People Mover im öffentlichen Personennahverkehr (ÖPNV). Im vorliegenden Teilprojekt wurden diese Aspekte im Hinblick auf die Verkehrssicherheit analysiert.

Um ein Projekt wie den Shuttlebus in Bad Birnbach durchzuführen, muss eine technische Prüfstelle hinzugezogen werden, die die Zulassungsempfehlung aussprechen kann. Hierbei kontrolliert die technische Prüfstelle die Einhaltung der geltenden Gesetze und achtet bei Ausnahmen darauf, dass die notwendige Sicherheit gewährleistet ist. Im Falle des Projektes in Bad Birnbach konnte nicht nur die Technik des Shuttlebusses als alleiniges System betrachtet werden. Es musste ein Gesamtkonzept freigegeben werden, das den Betrieb und das Umfeld mit einschließt. Hierbei müssen Hersteller, Betreiber, technische Prüfstelle, lokale Behörden und Landesbehörden zusammenarbeiten, bis eine Zulassung des Shuttlebusses ausgesprochen werden kann. Entsprechend dem Gesamtkonzept wurden Änderungen am Bus vorgenommen, Funktionen eingeschränkt, Infrastruktur angepasst und ein Operator, der durchgängig an Bord ist und notfalls eingreifen kann, eingesetzt. Hinzu kommt, dass der Bus auf einer festgelegten Route verkehrt, ohne diese verlassen zu können.

Hinsichtlich der Technik wurde nicht der Bus als Gesamtsystem betrachtet, sondern die Forschungen konzentrierten sich auf den Teil, der essenziell für einen autonomen Betrieb ist. Dieser setzt sich wiederum aus den Subsystemen Aktuatorik und Sensorik zusammen. Dabei ist die Aktuatorik für die Bewegung - beschleunigen, bremsen, lenken - des Busses zuständig. Die Sensorik ist für die Ansteuerung der Aktuatorik verantwortlich. Sie bildet sozusagen „den Kopf“ des Systems, der durch Sensoren das Umfeld erfasst und mittels Analysealgorithmen entscheidet, wie sich der Bus bewegt.

Für einen fahrerlosen Betrieb müssen die Orientierung an dem unveränderlichen sowie die Reaktionen auf den veränderlichen Bestandteil des Umfeldes durch die Sensorik sichergestellt sein. In den vorliegenden Forschungen wurde das Hauptaugenmerk auf die Interaktion mit weiteren Verkehrsteilnehmern und anderen Objekten sowie das daraus resultierende unfallfreie Fahren gelegt.

Der Bus in Bad Birnbach soll den Bahnhof mit dem Marktplatz verbinden und dabei an der Rottal Terme und dem Touristenzentrum Artrium halten. Zu Beginn der Forschungen verkehrte der Bus lediglich zwischen Marktplatz und Rottal Terme mit einem Halt am 
Artrium. Zur Mitte des Projekts wurde zusätzlich ein Teilstück der Strecke in Richtung des Bahnhofs in Betrieb genommen. Die Route führt über befahrene Straßen, Fußgängerzonen - zeitweise mit Lieferverkehr - und asphaltierte Feldwege. An verschiedenen Stellen mussten Veränderungen der Infrastruktur vorgenommen werden, um den Zulassungsbedingungen Rechnung zu tragen. Darunter finden sich Markierungen zur Orientierung sowie Straßenverbreiterungen wieder, die mit den Systemen des Busses abgestimmt wurden.

Im vorliegenden Teilprojekt wird in erster Linie die Frage behandelt, ob der Bus ohne die zusätzlich getroffenen Sicherheitsmaßnahmen einen unfallfreien Betrieb darstellen könnte. Zusätzlich werden weitere Erkenntnisse hinsichtlich des Verkehrsflusses und des Fahrkomforts erhofft. Mittels Dokumentationen durch die Operatoren und Interviews mit selbigen werden Informationen über Verkehrssituationen gesammelt, in denen die Fahrt des Busses unplanmäßig unterbrochen wird. Die Datenerhebung wird durch zufällige Gespräche mit den Fahrgästen sowie eigenen Erfahrungen ergänzt, aber bei der prozentualen Auswertung nicht berücksichtigt. Durch die Analyse der gesammelten Daten werden häufige Ursachen für die Unterbrechung der Fahrt sowie kritische Situationen, die der Bus unfallfrei nicht selbstständig durchfahren kann, identifiziert.

In einer abschließenden Versuchsreihe werden die Sicherheitseinrichtungen des Busses validiert sowie Gefahrensituationen nachgestellt. Hierdurch werden Sicherheitslücken aufgezeigt, die bei den weiteren Entwicklungen von autonomen Fahrzeugen berücksichtigt und abgesichert werden müssen. Es wird davon ausgegangen, dass die meisten Anforderungen alle Entwicklungen der autonomen Mobilität betreffen. Einige werden aber voraussichtlich spezielle Anforderungen für den Einsatz der People Mover im ÖPNV sein.

\subsection{Fahrzeugbeschreibung}

\subsubsection{Autonomer Shuttlebus}

Die bestehende Verkehrsinfrastruktur ist vielerorts und ganz besonders in Ballungsgebieten überlastet. Dies führt zu zeitlichen Verzögerungen, sinkender Verkehrseffizienz und volkswirtschaftlichen Kosten. Diese und viele weitere Herausforderungen haben dazu geführt, dass seit einiger Zeit intensiv an Technologien zum automatisierten Fahren sowie damit verbundenen neuen Mobilitätskonzepten gearbeitet wird. Dieses Thema betrifft nicht nur den Pkw-Verkehr, sondern insbesondere auch Nutzfahrzeuge sowie den Personentransport in Bussen.

In den Städten ist der ÖPNV ein wichtiger Baustein der Mobilität, jedoch lassen sich nicht alle Strecken wirtschaftlich betreiben. Die Automatisierung kann hier einen Beitrag zum kostengünstigeren Betrieb eines Busses liefern. Mithilfe fahrerloser U-Bahnen konnte man bereits Erfahrungen mit dem automatisierten Betrieb sammeln. Jedoch bestehen zu einem autonom fahrenden Bus zwei wesentliche Unterschiede. Zum einen wird eine U-Bahn in einem abgeschlossenen Verkehrsbereich betrieben, zum anderen ist eine Bahn natürlich schienengebunden. Auf Strecken mit einem geringen Passagieraufkommen sind 
dagegen autonom fahrende Shuttlebusse kostengünstiger, da keine spezielle Infrastruktur erforderlich ist. Solche Busse haben i. d. R. einen Elektroantrieb, was dann auch der Umweltsituation in Innenstädten zu Gute kommt.

Der erste autonome Shuttlebus, der in Deutschland im öffentlichen Straßenverkehr zugelassen ist, fährt seit Herbst 2017 im Kurort Bad Birnbach. Da der Begriff „Autonomer Shuttlebus" nicht klar definiert ist, muss zunächst der Automatisierungsgrad des Busses vom Typ EZ10 (Hersteller EasyMile) abgeklärt werden. Vor ein paar Jahren wurden dazu sowohl vom Verband der Automobilindustrie (VDA 2015) in Zusammenarbeit mit der Bundesanstalt für Straßenwesen (BASt) als auch von der Society of Automobile Engineers (SAE 2018) die Stufen der Automatisierung definiert (siehe auch VDI 2018).

Level/Stufe 0 (,No Driving Automation“/,Driver only“):

Der Fahrer erfüllt dauerhaft die gesamte Fahraufgabe selbstständig. Andere Fahrzeugsysteme können allerdings Warnungen anzeigen oder temporäre Unterstützung bieten. Beispiel hierfür ist ein ABS-System.

Level/Stufe 1 („Driver Assistance“/,Assistiert“):

Das System übernimmt dauerhaft die Längs- oder Querführung in einem bestimmten Operationsbereich. Der Fahrer verantwortet sowohl die jeweils andere Führung als auch die restlichen Teile der Fahraufgabe. Er hat das System jederzeit zu überwachen und einzugreifen, um ein sicheres Agieren des Fahrzeuges zu gewährleisten. Der Fahrer bestimmt, ob das System aktiviert werden soll und übernimmt die vollständige dynamische Fahraufgabe, sobald er es wünscht oder es verlangt wird. Beispiele hierfür sind das ACC-System (Abstandsregeltempomat) oder der Spurhalteassistent. So ist der Fahrer beim Spurhalteassistent für das Beschleunigen und Bremsen verantwortlich und wird hinsichtlich der Querführung vom System unterstützt.

Level/Stufe 2 („,Partial Driving Automation“/,Teilautomatisiert“):

Das System übernimmt dauerhaft die Längs- und Querführung in einem bestimmten Operationsbereich. Es gelten die gleichen Rollenverteilungen wie unter Stufe 1. Als Beispiele hierfür sind der unterstützende Stauassistent oder das sogenannte Schlüsselparken zu nennen. Zum Schlüsselparken lässt sich das Fahrzeug über den Autoschlüssel oder eine Handy-App von außen automatisch in eine vorher ausgewählte Parklücke ein- und anschließend wieder herausfahren. Der Fahrroboter übernimmt also die Quer- und Längsführung (Einparken) für kurze Zeit in einer spezifischen Situation (Parkvorgang). Level 2 ist durch eine dauerhafte Überwachung des Fahrers sowie die jederzeitige Bereitschaft zur vollständigen Übernahme gekennzeichnet.

Level/Stufe 3 (,Conditional Driving Automation“/,Hochautomatisiert“):

Das System übernimmt dauerhaft und vollständig die dynamische Fahraufgabe in einem bestimmten Operationsbereich. Der Fahrer entscheidet, ob das System aktiviert werden soll. Er muss das System nicht mehr kontinuierlich überwachen, muss aber innerhalb eines angemessenen Zeitraums eingreifen können, sobald das System dies verlangt oder Systemgrenzen auftreten. Darüber hinaus bestimmt er, ob und wie ein risikominimaler Zustand erreicht wird. Ein Beispiel hierfür ist ein Assistenzsystem, das auf Autobahnen zeitweise die Längs- und Querführung übernimmt. 
Level/Stufe 4 („High Driving Automation“/,Vollautomatisiert“):

Das System übernimmt dauerhaft und vollständig die Fahraufgabe in einem bestimmten Operationsbereich. Der Fahrer entscheidet, ob er das System nutzt oder ob er selbst fährt. Er muss nicht in der Lage sein, einzugreifen und muss nicht bestimmen, ob und wie ein risikominimaler Zustand erreicht wird. Das System erkennt Systemgrenzen und fordert notfalls den Fahrer auf, innerhalb eines angemessenen Zeitraums einzugreifen. Greift der Fahrer nicht ein, wird das Fahrzeug vom System in den risikominimalen Zustand überführt. Ab Stufe 4 wird der Fahrer auch als Nutzer bezeichnet.

Level/Stufe 5 (,Full Driving Automation“/,Fahrerlos“):

Das System übernimmt dauerhaft, vollständig und bedingungslos die Fahraufgabe. Es befindet sich kein Fahrer im Fahrzeug und das Fahrzeug ist nicht mehr an Einschränkungen durch einen Operationsbereich gebunden. Es gelten die gleichen Rollenverteilungen wie unter Stufe 4. Ein Beispiel hierfür ist ein fahrerloses Shuttle auf dafür vorgesehenen Strecken.

Abb. 5.1 zeigt die Stufen der Automatisierung nach VDA (2015), Abb. 5.2 nach SAE-Einteilung (SAE 2018).

Da ein autonomer Shuttlebus wie der EasyMile EZ10 theoretisch keinen Fahrer benötigt, wird das Fahrzeug ohne Lenkrad und Pedale ausgeliefert. Zur Absicherung bzw. zur Erfüllung der Zulassungsbedingungen wird allerdings jede Fahrt von einem Operator begleitet, der das Fahrzeug über Joystick und Tablet-Computer steuern kann. Falls das

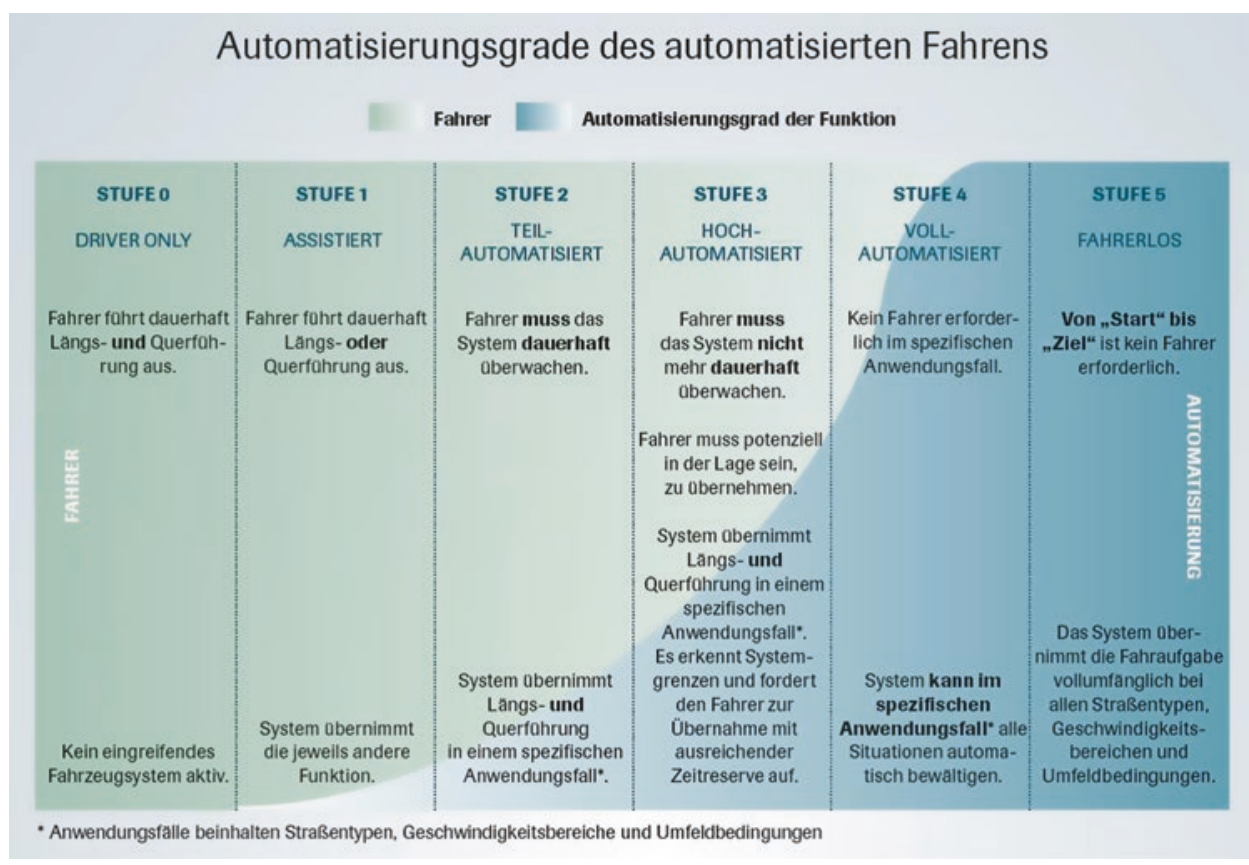

Abb. 5.1 Übersicht der Automatisierungsgrade nach VDA. (Quelle: VDA 2015) 


\begin{tabular}{|c|c|c|c|c|c|c|}
\hline \multirow[b]{2}{*}{ Ј } & \multirow[b]{2}{*}{ Name } & \multirow[b]{2}{*}{ Narrative definition } & \multicolumn{2}{|c|}{$D D T$} & \multirow[b]{2}{*}{$\begin{array}{c}D D T \\
\text { fallback }\end{array}$} & \multirow[b]{2}{*}{$O D D$} \\
\hline & & & $\begin{array}{c}\text { Sustained } \\
\text { lateral and } \\
\text { longitudinal } \\
\text { vehicle motion } \\
\text { control }\end{array}$ & OEDR & & \\
\hline \multicolumn{3}{|c|}{ Driver performs part or all of the $D D T$} & & & & \\
\hline 0 & $\begin{array}{l}\text { No Driving } \\
\text { Automation }\end{array}$ & $\begin{array}{c}\text { The performance by the driver of the entire } D D T \text {, even } \\
\text { when enhanced by active safety systems. }\end{array}$ & Driver & Driver & Driver & $\mathrm{n} / \mathrm{a}$ \\
\hline 1 & $\begin{array}{c}\text { Driver } \\
\text { Assistance }\end{array}$ & $\begin{array}{l}\text { The sustained and } O D D \text {-specific execution by a } \\
\text { driving automation system of either the lateral or the } \\
\text { longitudinal vehicle motion control subtask of the DDT } \\
\text { (but not both simultaneously) with the expectation that } \\
\text { the driver performs the remainder of the DDT. }\end{array}$ & $\begin{array}{l}\text { Driver and } \\
\text { System }\end{array}$ & Driver & Driver & Limited \\
\hline 2 & $\begin{array}{c}\text { Partial } \\
\text { Driving } \\
\text { Automation }\end{array}$ & $\begin{array}{c}\text { The sustained and } O D D \text {-specific execution by a } \\
\text { driving automation system of both the lateral and } \\
\text { longitudinal vehicle motion control subtask of the } \\
D D T \text { with the expectation that the driver completes } \\
\text { the OEDR subtask and supervises the } \\
\text { driving automation system. }\end{array}$ & System & Driver & Driver & Limited \\
\hline \multicolumn{3}{|c|}{ ADS ("System") performs the entire $D D T$ (while engaged) } & & & & \\
\hline 3 & $\begin{array}{l}\text { Conditional } \\
\text { Driving } \\
\text { Automation }\end{array}$ & $\begin{array}{l}\text { The sustained and ODD-specific performance by an } \\
A D S \text { of the entire DDT with the expectation that the } \\
D D T \text { fallback-ready user is receptive to } A D S \text {-issued } \\
\text { requests to intervene, as well as to DDT performance- } \\
\text { relevant system failures in other vehicle systems, and } \\
\text { will respond appropriately. }\end{array}$ & System & System & $\begin{array}{l}\text { Fallback- } \\
\text { ready user } \\
\text { (becomes } \\
\text { the driver } \\
\text { during } \\
\text { fallback) }\end{array}$ & Limited \\
\hline 4 & $\begin{array}{c}\text { High } \\
\text { Driving } \\
\text { Automation }\end{array}$ & $\begin{array}{c}\text { The sustained and } O D D \text {-specific performance by an } \\
A D S \text { of the entire } D D T \text { and } D D T \text { fallback without any } \\
\text { expectation that a user will respond to a } \\
\text { request to intervene. }\end{array}$ & System & System & System & Limited \\
\hline 5 & $\begin{array}{c}\text { Full } \\
\text { Driving } \\
\text { Automation }\end{array}$ & $\begin{array}{l}\text { The sustained and unconditional (i.e., not } O D D \text { - } \\
\text { specific) performance by an } A D S \text { of the entire } D D T \\
\text { and } D D T \text { fallback without any expectation that a user } \\
\text { will respond to a request to intervene. }\end{array}$ & System & System & System & Unlimited \\
\hline
\end{tabular}

Abb. 5.2 Automatisierungsgrade nach SAE-Definition. (Quelle: SAE 2018)

System an Grenzen stößt, kann der Operator bremsen, beschleunigen und lenken. Der Shuttlebus könnte - da ohne Lenkrad und Pedale ausgerüstet und aufgrund des symmetrischen Erscheinungsbilds - theoretisch ohne Wenden in beide Richtungen fahren, was jedoch in Bad Birnbach in der Typgenehmigung ausgeschlossen werden musste.

Der Begriff „Autonomer Shuttlebus“ impliziert, dass es sich um ein Fahrzeug der Stufe 5 nach SAE handeln müsste. Level 5 sieht vor, dass das System dauerhaft, vollständig und bedingungslos die Fahraufgabe übernimmt. Dazu gehört auch das Bewältigen aller Straßenbedingungen, Umgebungen, Verkehrssituationen und Geschwindigkeitsbereiche. Die Strecken, auf denen der EZ10 in Bad Birnbach verkehrt, werden hochgenau über Differential-GPS (dGPS) in das Fahrzeug einprogrammiert, weshalb es auf virtuellen Schienen fährt. Außerdem weiß der Bus auf dieser Strecke jederzeit, wo er sich befindet und wo er entlangzufahren hat. Das Fahrzeug kann sich so aber nur innerhalb vorher definierter Bereiche bewegen. Es kann Objekte wahrnehmen, aber nicht eigenständig 
umfahren. Ebenso ist eine Freigabe des Operators beim Linksabbiegen erforderlich. Darüber hinaus liegt die Operationsgeschwindigkeit bei maximal $15 \mathrm{~km} / \mathrm{h}$. Demnach sind die Kriterien für Level 5 nicht erfüllt. Dies wird auch durch die Tatsache gestützt, dass sich im Shuttlebus ein Operator befinden muss, der durchgängig die Fahrt überwacht und jederzeit eingreifen kann.

Zusammenfassend lässt sich sagen, dass der Shuttlebus aufgrund der beschriebenen Eigenschaften als ein Fahrzeug der Stufe 2 eingeordnet werden muss. Das System übernimmt zwar die Längs- und Querführung des Fahrzeuges und erkennt seine Grenzen, muss aber gemäß Zulassungsvorgaben ständig vom Operator überwacht werden. Um als Level-3-Fahrzeug eingestuft zu werden, müsste die ständige Überwachung des Systems durch den Operator entfallen und das Fahrzeug nur im Fehlerfall den Operator zur Übernahme auffordern. Dies stellt den grundlegenden Unterschied zu Stufe 2 dar.

Natürlich ist auch der Betrieb eines Level-2-Fahrzeuges im öffentlichen Straßenverkehr bestens geeignet, Erfahrungen und Daten zur Erhöhung der Verfügbarkeit, der Zuverlässigkeit und der Sicherheit zu sammeln.

\subsubsection{Technik, Funktionsweise und Betrieb des Shuttlebusses}

Das in Bad Birnbach eingesetzte Shuttle EZ10 ist ein autonomer, fahrerloser Kleinbus der Firma EasyMile. Der Bus besitzt eine Kapazität für 12 Personen (6 Sitz- und 6 Stehplätze ohne Sicherheitsgurt). Die Fahrtgeschwindigkeit beträgt bis zu $40 \mathrm{~km} / \mathrm{h}$. Angetrieben wird der Bus von einem Asynchron-Drehstrommotor. Dieser bezieht seine Energie aus Akkus, welche bis zu 14 Stunden autonomes Fahren ermöglichen.

Um den autonomen Betrieb zu ermöglichen, verfügt der Bus über eine Lokalisierung zur Positionsbestimmung und eine Hinderniserkennung.

\subsubsection{Lokalisierung}

Hier greifen verschiedene Messmethoden und Sensoren ineinander, um eine möglichst genaue Positionsbestimmung zu ermöglichen. Die Odometrie verwendet Raddrehzahlsensoren und eine verbaute Inertial Measurement Unit (IMU) zur Lageschätzung. Auf dem Dach des Busses sind zwei Lidarsensoren jeweils nach vorne und rückwärts gerichtet mit $110^{\circ}$ Öffnungs- und 3,2 Neigungswinkel verbaut (Abb. 5.3). Weiter verfügt der Bus über dGPS, welches die nötigen Korrekturdaten über ein verbautes Mobilfunkmodem erhält.

Abb. 5.3 Sichtfeld Multi-

LIDAR zur

Positionsbestimmung. (Quelle:

EasyMile o. J.)

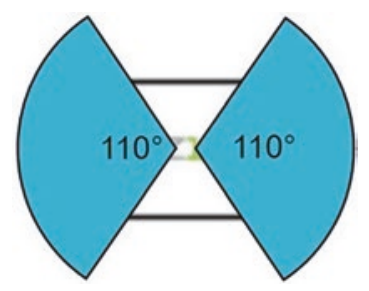


Die Odometrie-, LIDAR- und dGPS-Daten werden zusammengeführt und abgeglichen, um so die Position möglichst exakt bestimmen zu können. Diese IST-Positionsdaten werden im letzten Schritt per Simultaneous Localization and Mapping, kurz SLAM-Verfahren, mit den im Bus statisch gespeicherten Kartendaten abgeglichen.

\subsubsection{Hinderniserkennung}

$\mathrm{Da}$ in Bad Birnbach zwei Busse mit verschiedenen Technikständen Generation 1 Version 2.0 und Version 2.3 (Gen1 2.0 und 2.3) eingesetzt werden, unterscheidet sich die Anzahl und die Art der verwendeten Sensoren zur Objekterkennung. Die ältere Version 2.0 verwendet vier einstrahlige Lidarsensoren mit einem Öffnungswinkel von $270^{\circ}$, welche an den Ecken des Shuttles auf $30 \mathrm{~cm}$ Höhe angebracht sind (Abb. 5.4).

Die neuere Version 2.3 des Busses verwendet zusätzlich zwei Multi-Lidarsensoren, welche jeweils nach vorne und rückwärts gerichtet mit $180^{\circ}$ Öffnungs- und $32^{\circ}$ Neigungswinkel verbaut sind (Abb. 5.5).

Die Darstellung in Abb. 5.6 fasst die genannten Sensoren zusammen, welche im Bus verwendet werden. In Grau abgebildet sind zwei Kameras, die jeweils vorder- und rückseitig auf ca. 2 m Höhe angebracht sind. Diese sind im aktuellen Technikstand des Busses außer Betrieb und werden daher nicht weiter berücksichtigt.

Durch die vier einstrahligen LIDAR und das Multi-LIDAR-System werden um den Bus zwei Perimeter d1 und d2 errichtet (Abb. 5.7). Wenn ein Objekt in den Perimeter d2 eintritt und erkannt wird, verlangsamt der Bus seine Fahrt. Bei Abnahme des Abstandes

Abb. 5.4 Sichtfeld einstrahlige LIDAR. (Quelle: EasyMile o. J.)

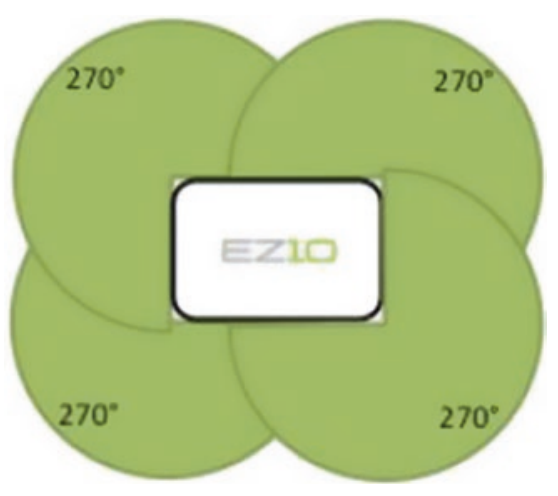

Abb. 5.5 Sichtfeld MultiLIDAR zur Hinderniserkennung. (Quelle: EasyMile o. J.)

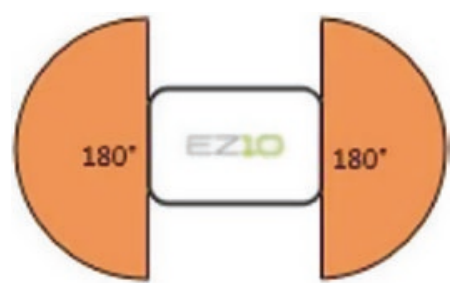


Abb. 5.6 Zusammenfassung aller Sensoren. (Quelle: EasyMile o. J.)
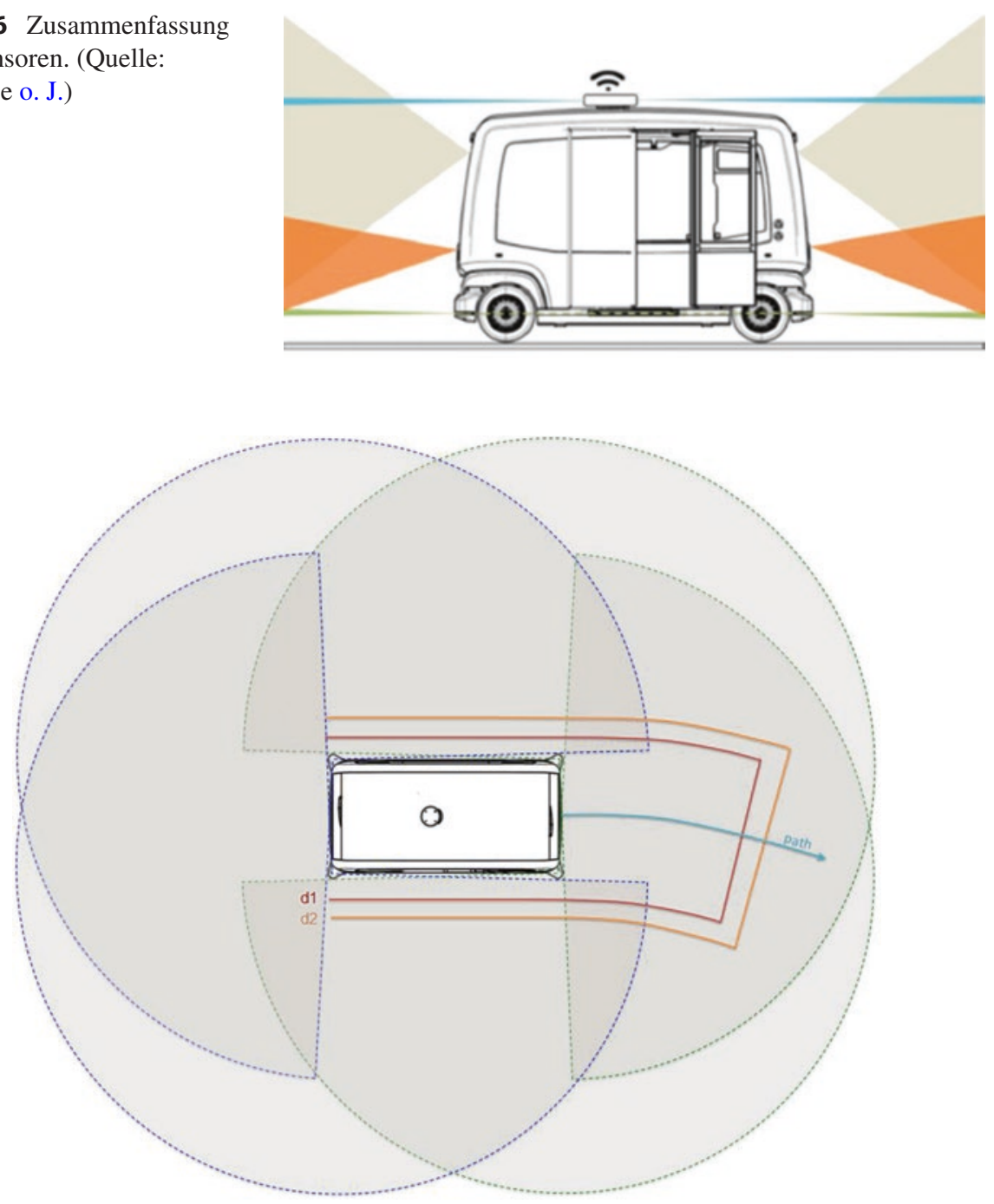

Abb. 5.7 Darstellung Perimeter zur Objekterkennung. (Quelle: EasyMile o. J.)

zum Bus verringert sich die Geschwindigkeit bis auf $1 \mathrm{~km} / \mathrm{h}$ bei Erreichen von Perimeter d1. Beim Eintritt des Hindernisses in Perimeter d1 löst der Shuttlebus selbständig einen Not-Stopp aus und kommt unmittelbar zum Stehen. Abhängig davon, von welcher Seite das Hindernis auf den Bus zukommt, variieren die Abmessungen von d1 und d2. Seitlich beträgt der Abstand, laut Herstellerangabe, von der äußersten Kante des Shuttles zum Perimeter d1 $30 \mathrm{~cm}$ und zum Perimeter d2 $150 \mathrm{~cm}$. Hinter dem Shuttle gibt es keinen Perimeter d1 und d 2 und somit auch keine Reaktion auf Hindernisse. Vor dem Shuttle passen sich beide Perimeter dynamisch der Geschwindigkeit und Lenkbewegung an, wie in Abb. 5.7 dargestellt. 


\subsubsection{Zulassungsbedingungen für den Betrieb auf öffentlichen Straßen}

Das Fahren auf öffentlichen Straßen als selbstfahrendes Fahrzeug erforderte einen umfangreichen Prüf- und Zulassungsprozess. Durch die Zusammenarbeit von Hersteller, Betreiber, technischer Prüfstelle und Behörden wurden Ausnahmegenehmigungen ausgesprochen, ein Sicherheitskonzept entwickelt und Infrastrukturänderungen ausgearbeitet. In den Dokumenten ist eine Reihe von Auflagen gefordert:

- Funktionale Sicherheit

- Risiko und Gefährdungsanalyse in Anlehnung an ISO 26262-2011

- Risikobewertung von Szenarien nach Häufigkeit der Situation, Gefährdungsgrad und Beherrschbarkeit von Fehlern

- Ableitung von Maßnahmen zur Risikoverminderung als Teil des Sicherheitskonzepts

- Dynamische Tests zur Überprüfung des Sicherheitskonzepts

- Bus folgt automatisiert der Route, die einmalig per Laserscanner im Bordcomputer eingelesen wurde

- Geschwindigkeit auf $15 \mathrm{~km} / \mathrm{h}$ und Personenbeförderungsanzahl auf sechs begrenzt

- Betriebssicherheit

- Dynamische Fahrzeugtests zur Überprüfung von

- Bremssystem

- Lenksystem

- Autonome Fahrfunktionen

- Elektrische Sicherheit

- Elektromagnetische Verträglichkeit

- Keine Fahrten bei extremem Wetter wie Regen, Nebel oder Schnee

- Einstufung als SO.KFZ bis max. $25 \mathrm{~km} / \mathrm{h}$

- Wiener Übereinkommen über Straßenverkehr

- Beherrschungsgrundsatz: Fahrer muss jederzeit Kontrolle über das Fahrzeug haben

- Implikation: Fahrtbegleiter (Operator) an Bord als Voraussetzung, der jederzeit bei Bedarf in das Fahrgeschehen eingreifen kann

- Infrastruktur (bauliche Auflagen für die Strecke)

- Einbau von drei Rüttelschwellen, um die Geschwindigkeit des Verkehrs zu drosseln

- Fahrbahnverbreiterung auf zwei Abschnitten und Anbringen eines Mittelstreifens

- Anbringen von Hinweisschildern, um auf den autonomen Kleinbus aufmerksam zu machen (ioki o. J.)

- Aufstellen von Orientierungstafeln zur Lokalisierung bei wenig bebauten Gebieten

Erst nach Erfüllung und Umsetzung aller Auflagen wurde die Freigabe für das Gesamtsystem ausgesprochen und der Bus zum Betrieb auf öffentlichen Straßen zugelassen.

\subsubsection{Betrieb des Shuttlebusses und Streckenverlauf}

Ziel des Projektes ist es, den Marktplatz mit dem Bahnhof Bad Birnbach durch regulären Fahrgastbetrieb zu verbinden (Abb. 5.8). Die Strecke wurde in drei Abschnitte unterteilt, 


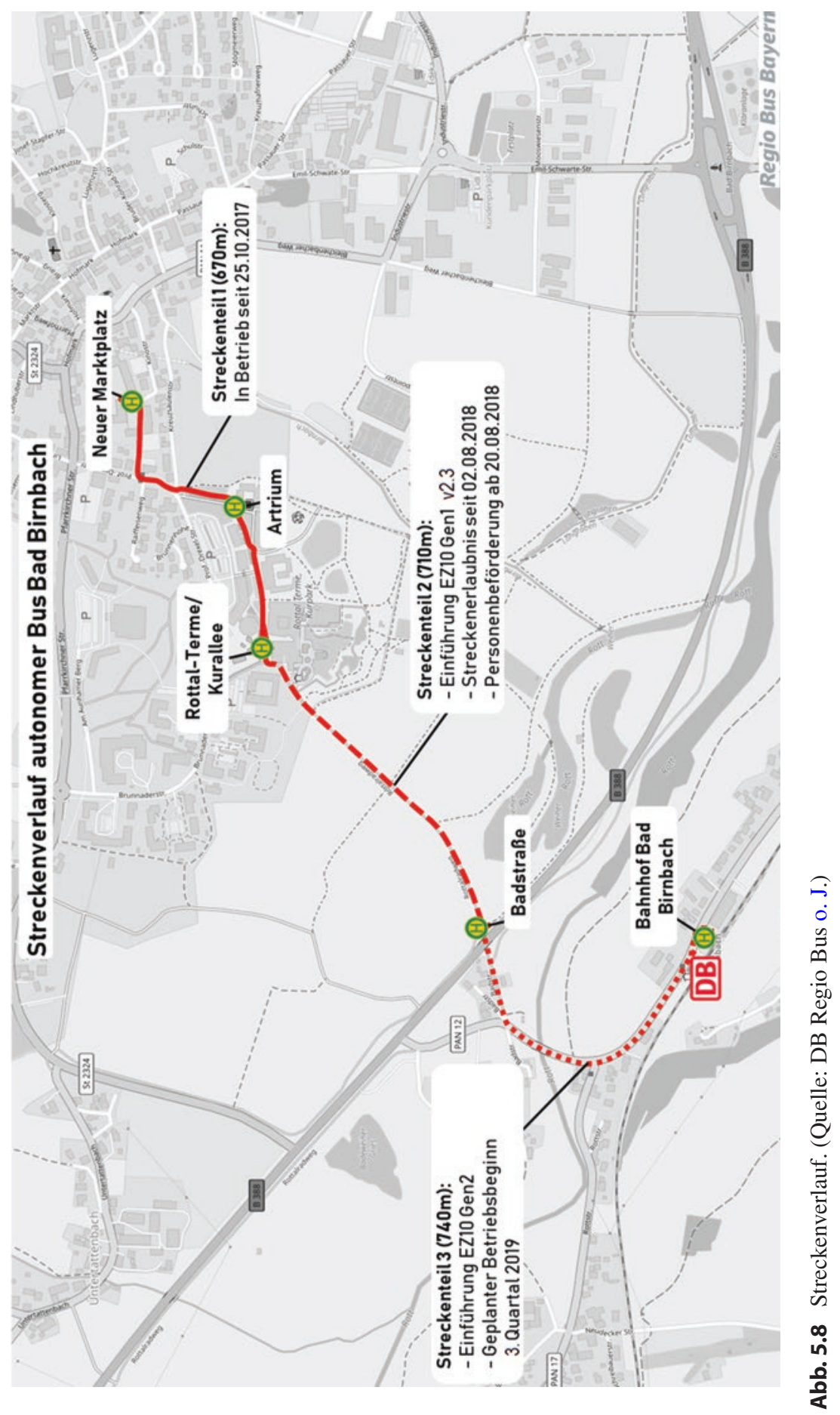


welche schrittweise in Betrieb genommen werden. Streckenabschnitt 1 mit 670 m Länge führt vom Marktplatz über das Artrium bis zur Rottal Terme. Der Betrieb startete am 25.10.2017. Der Bus fährt hier mit $10 \mathrm{~km} / \mathrm{h}$ von 8-18 Uhr an sieben Tagen der Woche im 20-Minuten-Takt. In diesem Abschnitt gibt es drei Haltestellen (Marktplatz, Artrium, Rottal Terme). Streckenabschnitt 2 führt von der Rottal Terme Richtung Bundesstraße B388 bis zur Haltestelle Badstraße kurz vor der Unterführung. Für diesen Streckenabschnitt wurde am 02.08.2018 die Zulassungserlaubnis erteilt und am 20.08.2018 mit der Personenbeförderung begonnen. Mit Inbetriebnahme des $710 \mathrm{~m}$ langen Abschnittes wurde auch der Bus gegen die überarbeitete Version 2.3 getauscht. Der neue Bus übernimmt damit den regulären Fahrbetrieb. Die alte Version 2.0 dient als Ersatz. Der dritte Streckenabschnitt führt von der Haltestelle Badstraße bis zum Bahnhof Bad Birnbach. Er misst eine Länge von 740 m. Der geplante Betriebsbeginn ist für das dritte Quartal 2019 vorgesehen. Hier soll auch die zweite Generation des EZ10 Shuttles erstmalig eingesetzt werden.

An allen Haltestellen hält der Bus selbstständig. Die Wiederaufnahme der Fahrt muss durch den Operator eingeleitet werden. Hierdurch wird unter anderem sichergestellt, dass sämtliche Fahrgäste ein- und ausgestiegen sind sowie die Strecke frei ist. An Stellen, an denen der Shuttlebus links abbiegen muss, schreiben die Zulassungsauflagen einen Stopp des Busses und die Freigabe durch den Operator vor. Auf der Strecke vom Marktplatz bis zum Artrium gibt es hiervon zwei Stellen, in der Gegenrichtung eine.

\subsubsection{Vergleichbare Projekte}

\subsubsection{1 Übersicht autonomer Kleinbusse}

Da das Thema der autonom fahrenden Kleinbusse recht neu ist, ist die Situation am Markt verfügbarer Fahrzeuge dieser Art überschaubar. Zwei Firmen haben sich in Europa am Markt etabliert, deren Kleinbusse für Projekte dieser Art verwendet werden. Hierbei handelt es sich um das Unternehmen EasyMile, von welchem auch für dieses Projekt Fahrzeuge genutzt werden, sowie das Unternehmen Navya. Beide Firmen sind in Frankreich ansässig und wurden im Jahre 2014 gegründet. Beide haben jeweils einen autonom fahrenden Kleinbus am Markt. In Tab. 5.1 und Tab. 5.2 sind die Unterschiede und Gemeinsamkeiten dargestellt, um einen Überblick zu geben.

Tab. 5.1 Basisdaten der Shuttlebusse von EasyMile und Navya (eigene Darstellung)

\begin{tabular}{l|l|l}
\hline Basisdaten & EasyMile EZ10 & Navya Arma \\
\hline Fahrgäste & 6 Sitz- und 6 Stehplätze & 11 Sitz- und 4 Stehplätze \\
\hline Abmessungen $(\mathrm{L} \times \mathrm{B} \times \mathrm{H})$ & $3,93 \times 1,99 \times 2,75 \mathrm{~m}$ & $4,75 \times 2,11 \times 2,65 \mathrm{~m}$ \\
\hline Gewicht $($ Leer/Gesamt) & $1800 / 2800 \mathrm{~kg}$ & $2400 / 3450 \mathrm{~kg}$ \\
\hline Zul. Gesamtgewicht & $2800 \mathrm{~kg}$ & $3450 \mathrm{~kg}$ \\
\hline Max. Geschwindigkeit & $40 \mathrm{~km} / \mathrm{h}$ & $25 \mathrm{~km} / \mathrm{h}$ \\
\hline Max. Fahrdauer & $14 \mathrm{~h}$ & $9 \mathrm{~h}$ \\
\hline
\end{tabular}


Tab. 5.2 Umfeldsensoren und deren Funktionen der Shuttlebusse von EasyMile und Navya (eigene Darstellung)

\begin{tabular}{|c|c|c|}
\hline Sensor & EasyMile EZ10 & Navya Arma \\
\hline Odometrie & Raddrehzahlsensor, IMU (Lokalisierung) & $\begin{array}{l}\text { Raddrehzahlsensor, IMU } \\
\text { (Lokalisierung) }\end{array}$ \\
\hline dGPS & $\begin{array}{l}\text { GPS + Korrektur Signal über Mobilfunk } \\
\text { (Lokalisierung) }\end{array}$ & GNSS RTK (Lokalisierung) \\
\hline LIDAR & $\begin{array}{l}2 \times 110^{\circ} \text { Multi-LIDAR (Lokalisierung) } \\
4 \times 270^{\circ} \text { Einstrahl-LIDAR, } 2 \times 180^{\circ} \\
\text { Multi-LIDAR (Objekterkennung) }\end{array}$ & $\begin{array}{l}2 \times 360^{\circ} \text { Multi-LIDAR, } 6 \times 180^{\circ} \\
\text { Einstrahl-LIDAR } \\
\text { (Lokalisierung und } \\
\text { Objekterkennung) }\end{array}$ \\
\hline Kamera & 2 (Objekterkennung) & 2 (Objekterkennung) \\
\hline Radar & nicht verbaut & nicht verbaut \\
\hline
\end{tabular}

Beide Fahrzeuge verwenden zur Lokalisierung Odometrie-, dGPS- und LIDAR-Daten. Zur Objekterkennung werden Kamera und ebenfalls LIDAR-Daten genutzt. Im Kleinbus von Navya werden zusätzlich vier Radarsensoren zur Objekterkennung eingesetzt.

\subsubsection{Vergleichbare Projekte im ÖPNV}

Projekte mit vergleichbarer Ausrichtung gibt es (Stand Januar 2019) auf der ganzen Welt. Zum Zweck der Vergleichbarkeit sind an dieser Stelle zwei Projekte in Deutschland mit ähnlicher Ausrichtung und Umfang aufgeführt.

\section{Berlin - Forschungsprojekt, ,Stimulate“6}

Die Berliner Verkehrsbetriebe und das Universitätsklinikum Charité testen seit Anfang 2018 zusammen den Einsatz von vier autonomen Kleinbussen des Anbieters Navya und EasyMile auf den Geländen Berlin-Mitte und Berlin-Wedding der Charité. Die beiden Standorte eignen sich besonders gut für das Projekt, da sie vom öffentlichen Straßennetz abgegrenzt sind. Trotzdem wird aufgrund der vielen Wege, Kreuzungen und Verkehrsteilnehmer das normale Verkehrsgeschehen wirklichkeitsnah abgebildet. Hier sind die Busse wochentags von 9-16 Uhr auf drei Rundkursen mit 26 Haltestellen und max. 20 km/h unterwegs (Neumann 2018). Vorerst wird analog zu Bad Birnbach mit einem Operator an Bord gefahren, der jederzeit eingreifen kann. Ziel von Stimulate ist das Sammeln von Erkenntnissen zur Akzeptanz der Nutzer sowie die Erprobung der technischen Umsetzung im Alltagsbetrieb (Krempl 2018).

\section{Hamburg - Forschungsprojekt „HEAT“6}

Das Projekt Hamburg Electric Autonomous Transportation, kurz HEAT, wurde von Partnern aus Stadt, Industrie und Forschung 2018 ins Leben gerufen. Ziel ist es, herauszufinden, ob sich autonome Kleinbusse für den Betrieb im ÖPNV eignen und ob die zugrunde liegende Technik robust genug ist, alle alltags- und sicherheitstechnischen Anforderungen zu erfüllen. HEAT startete 2018 mit der Planung und soll bis 2021 laufen. In Phase I und II wird noch ein Operator mitfahren, um eingreifen zu können. Ab Phase III, welche für 2021 
geplant ist, soll das Fahrzeug vollautonom mit bis zu $50 \mathrm{~km} / \mathrm{h}$ ohne Operator fahren. Der verwendete Kleinbus wird von der Firma IAV entwickelt. Er soll ähnlich wie die Busse von EasyMile und Navya mit Kameras, LIDAR und Radar ausgestattet sein. Zusätzlich soll hier V2X-Kommunikation eingesetzt werden, um die Sicherheit weiter zu erhöhen. Die Teststrecke wird 3,6 km durch die Hamburger Hafen City führen und über neun Haltestellen verfügen. Die Teststrecke soll - wie in Bad Birnbach - schrittweise eingeführt werden. Die V2X-Kommunikation wird in der finalen Ausbaustufe zwischen zwölf Ampeln und dem Kleinbus stattfinden. Die Strecke wurde ausgewählt, weil es sich ana$\log$ zu Berlin um ein räumlich abgegrenztes Gebiet mit vielen Bussen, Pkw sowie Fußgängern und Radfahrern handelt, welches das Verkehrsgeschehen realitätsnah abbildet (Gängrich 2018).

\subsection{Forschungsprojekt Technik}

\subsubsection{Projektablauf}

Das Projekt wurde in fünf Abschnitte untergliedert. Im ersten Abschnitt (Phase 1) wurden die Dokumentationsbögen für den Operator definiert. Die Kernpunkte waren hier die Entwicklung konkreter Fragestellungen mit dem Ziel einer präzisen Dokumentation für die Ursachenforschung. Es wurde die Sachlage der vorhandenen Dokumentation eruiert und deren Einbindung geprüft. Entscheidende Faktoren wurden identifiziert, charakterisiert und kategorisiert. Als letzter Punkt stand die Erfassung der Strecke mittels Kamera an. Ziel der ersten Phase war die Erstellung eines Dokumentationsbogens und die Definition des Reportings.

Im zweiten Abschnitt (Phase 2.1) stand die Analyse und Auswertung der Dokumentationsbögen an. Es wurden die Kameradaten und Dokumentationsbögen gesichtet und Interviews mit den Operatoren geführt, um anschließend die dokumentierten Situationen zu filtern und Gemeinsamkeiten in der Auswertung zu identifizieren. Das Zusammentragen aller relevanten Daten und das Clustern von kritischen Ereignissen waren die Ziele dieser Phase.

Als nächstes sollten im dritten Abschnitt (Phase 2.2) die Versuchsanordnungen definiert werden. Hierfür wurden die kritischen Ereignisse in Cluster zusammengefasst und für jedes Cluster eine Versuchskonfiguration erstellt. Es wurden nur Versuchskonfigurationen erstellt, welche reproduzierbar waren.

Im vierten Abschnitt (Phase 2.3) wurde die zuvor festgelegte Versuchskonfiguration in Bad Birnbach unter realen Bedingungen getestet. Hierfür wurden verschiedene Hindernisse sowie Fußgänger- und Fahrzeugattrappen verwendet, um kritische Situationen nachzustellen. So konnten gefahrlos technische Grenzen des Busses ausgelotet werden.

Im letzten Abschnitt (Phase 3) standen die Auswertung der Daten und der Projektbericht an. Die Versuchsergebnisse wurden unter Beschreibung der Einflussfaktoren, aufgetretener Fehler sowie der Betrachtung von Ursachen und Situationen analysiert. Es wurden Auswirkungen auf den Streckenverlauf untersucht und die Anforderungen an das Lastenheft geprüft. Im letzten Schritt wurde der Abschlussbericht erstellt. 


\subsubsection{Durchführung und Ergebnisse der Projektphasen}

\subsubsection{IST-Stand zu Projektbeginn}

Zu Projektbeginn wurde die Sachlage der aktuellen Dokumentation eruiert (Phase 1), um zu prüfen, welche Daten schon erfasst wurden und ob diese weiterverwendet werden konnten. Es wurde festgestellt, dass die Operatoren nur technische Probleme des Busses dokumentierten (z. B. Scheibenwischer defekt, Tür klemmt beim Öffnen).

Die Strecke sowie deren Ausbaustufen wurden in Abschn. 5.2.2.4 bereits näher erläutert und in Abb. 5.8 dargestellt. In Abschn. 5.2.2.3 wurden die geforderten baulichen Änderungen, welche an der Straße durchgeführt werden mussten, ebenfalls bereits aufgeführt. Zusammenfassend gesagt, ist zu Projektbeginn die erste Ausbaustufe der Strecke in Betrieb. Die zweite Ausbaustufe wurde während der Projektphase 2 in Betrieb genommen. An der Strecke wurden drei bauliche Veränderungen vorgenommen, um den Anforderungen der Betriebszulassung zu genügen (siehe Abschn. 5.2.2.3 Abschnitt Infrastruktur).

\subsubsection{Phase 1 - Definition der Dokumentationsbögen}

Die Idee hinter den Dokumentationsbögen war, möglichst viele kritische Ereignisse zu dokumentieren, um die für Phase 2.2 nötigen Versuchskonfigurationen erstellen zu können. Weiter war es wichtig, die Anforderungen an die Operatoren möglichst gering zu halten, da hier der Zeitfaktor eine entscheidende Rolle spielte. Deshalb sollten die Dokumentationsbögen intuitiv und schnell ausfüllbar sein. Die Operatoren wurden angehalten, die Bögen möglichst unmittelbar nach einer kritischen Situation auszufüllen und die Daten möglichst unzensiert niederzuschreiben.

Der Dokumentationsbogen (Abb. 5.9) wurde in zwei Bereiche untergliedert. Der obere Bereich enthält drei Bilder von Kreuzungen ( $\mathrm{A}=$ Marktplatz, $\mathrm{B}=$ Mitte, $\mathrm{C}=$ Artrium), ein Bild mit der kompletten Streckenübersicht und ein Piktogramm des Busses. In den Bildern sollte vom Operator eingetragen werden, an welchem Ort auf der Strecke sich die kritische Situation ereignete. Die drei Kreuzungen wurden hierbei als Brennpunkt für kritische Situationen vermutet und deshalb ausgewählt. Weiter sollte der Hergang vom Operator eingezeichnet werden, damit dieser bei der Auswertung nachvollzogen werden kann. In dem Piktogramm sollte die Richtung des Hindernisses im Verhältnis zum Bus eingezeichnet werden.

Der untere Bereich enthält die Basisdaten (Operatorname, Datum, Uhrzeit). Weiter wird die konkrete Situation abgefragt (Not-Stopp, Soft-Stopp, Komfortsituation) und ob diese durch den Operator oder das Shuttle ausgelöst wurde.

- Not-Stopp (Shuttle hält sofort an $\rightarrow$ Vollbremsung $\rightarrow$ kritische Situation)

- Soft-Stopp (Shuttle hält allmählich an $\rightarrow$ normaler Bremsvorgang $\rightarrow$ mögliche kritische Situation)

- Komfortsituation (Shuttle verlangsamt, hält nicht an $\rightarrow$ sanfter Bremsvorgang $\rightarrow$ keine kritische Situation)

Es werden ebenfalls Daten zu Fahrtrichtung, Wetter, Hindernis Geschwindigkeit und Art sowie Fahrzeug-ID abgefragt. 


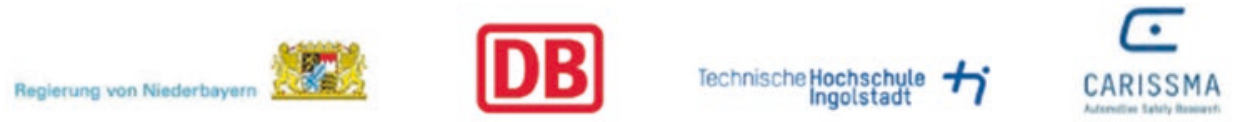

\section{Dokumentation außergewöhnlicher Verkehrssituationen}
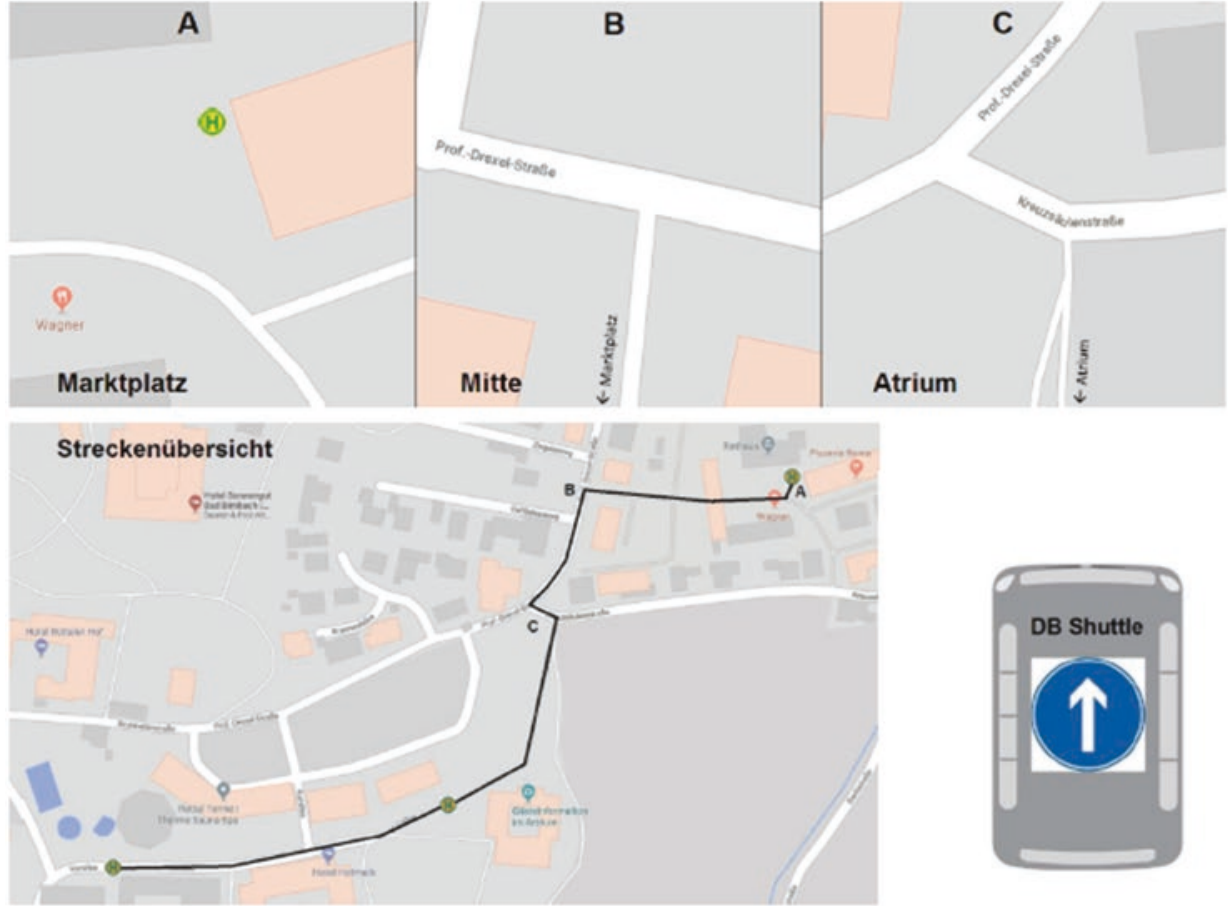

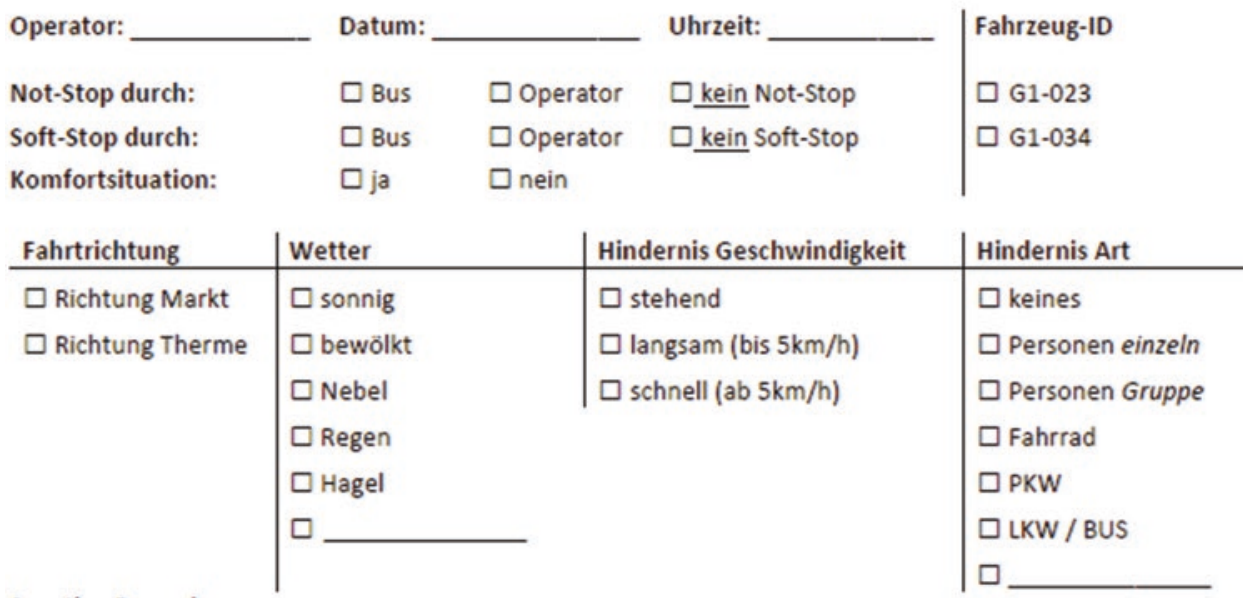

Sonstige Bemerkungen:

Abb. 5.9 Dokumentationsbogen (eigene Darstellung) 
Alle Daten im unteren Teil des Dokumentationsbogens - außer den Basisdaten - werden durch das Ankreuzen vorgegebener Antworten erfasst.

\subsubsection{Phase 2.1 - Analyse der Dokumentationsbögen}

Im Zeitraum vom 21.07.2018 bis 19.11.2018 wurden die Dokumentationsbögen der Operatoren gesichtet und ausgewertet. In Summe sind hier 304 Datensätze eingegangen. Die Ergebnisse werden im Folgenden als Ganzes in einem generellen Überblick und nach vereinzelten besonderen Vorkommnissen bewertet.

Abb. 5.10 zeigt die Fahrstrecke unterteilt nach Abschnitten (A, AB, B, BC, C, C1, C2 und C3). Der Marktplatz (A) und die Fußgängerbereiche, vom Einzelhandel geprägte Strecke durch die Innenstadt $(\mathrm{AB})$ als auch die Ortsstraße $(\mathrm{BC})$ können von Fußgängern und Kraftfahrzeugen gleichermaßen passiert werden. Der Bereich $\mathrm{C}$ hingegen markiert die Ein- und Ausfahrt in die Fußgängerzone (Kurallee), welche sich in Bereiche C1 und C2 unterteilt. Diese Bereiche sind von privaten Kraftfahrzeugen ausgeschlossen und als Fußgängerzone deklariert (Lieferverkehr frei). Der Bereich C3 ist ebenfalls Pkw-frei und nur für landwirtschaftliche Fahrzeuge freigegeben.

Auf der kurzen Strecke von ca. 1,5 km (von A bis Ende C3) kann es, durch die variablen Begebenheiten der Strecke, schnell zu unterschiedlichen Arten von Hindernissen kommen (Definition Hindernis: Bus stoppt bzw. verlangsamt seine reguläre Fahrt). Die Operatoren haben hierzu in den Dokumentationsbögen jeweils Stellung genommen und die Hindernis-

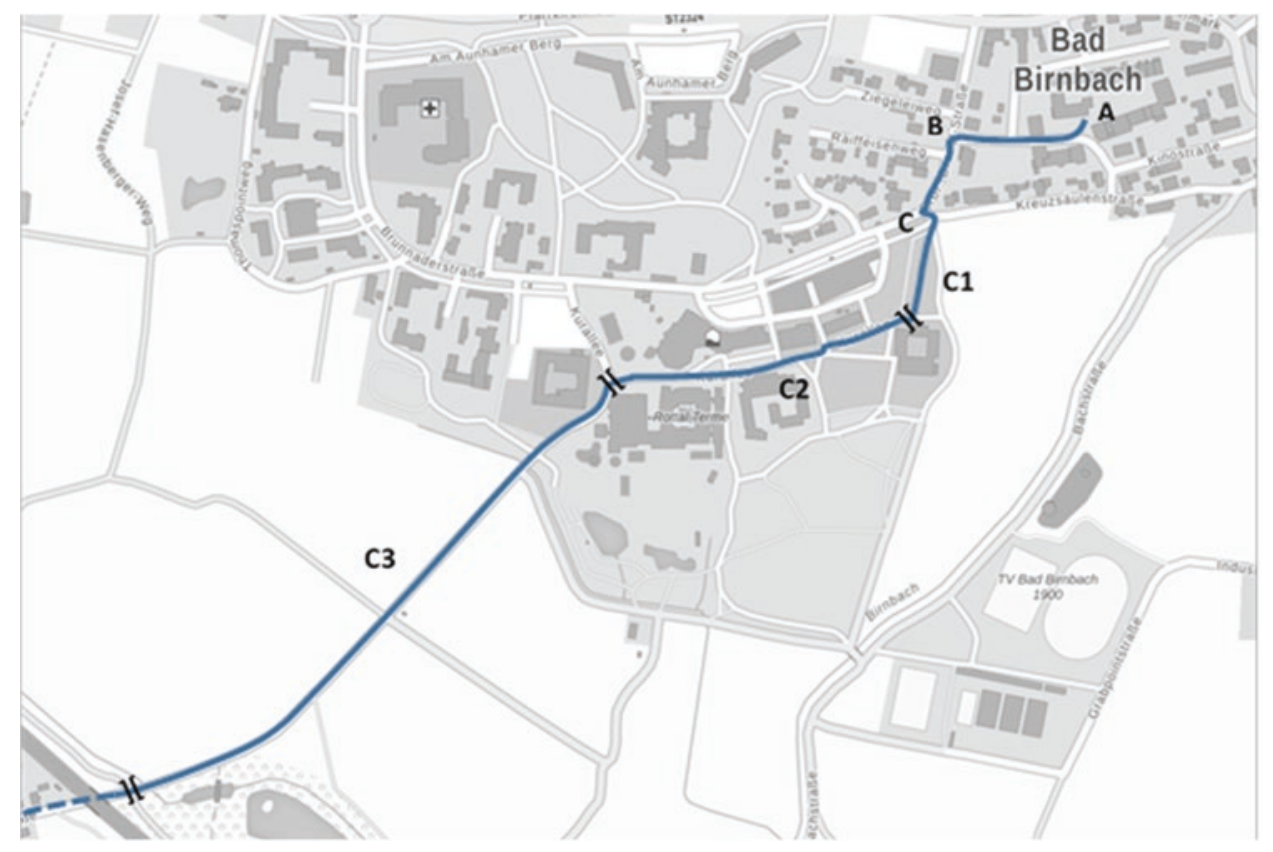

Abb. 5.10 Streckenverlauf des Shuttlebusses in Bad Birnbach (eigene Darstellung) 
Abb. 5.11 Einteilung der Hindernisse nach ihrer Art (eigene Darstellung)
Abb. 5.12 Einteilung der Hindernisse nach ihrer Geschwindigkeit (eigene Darstellung)
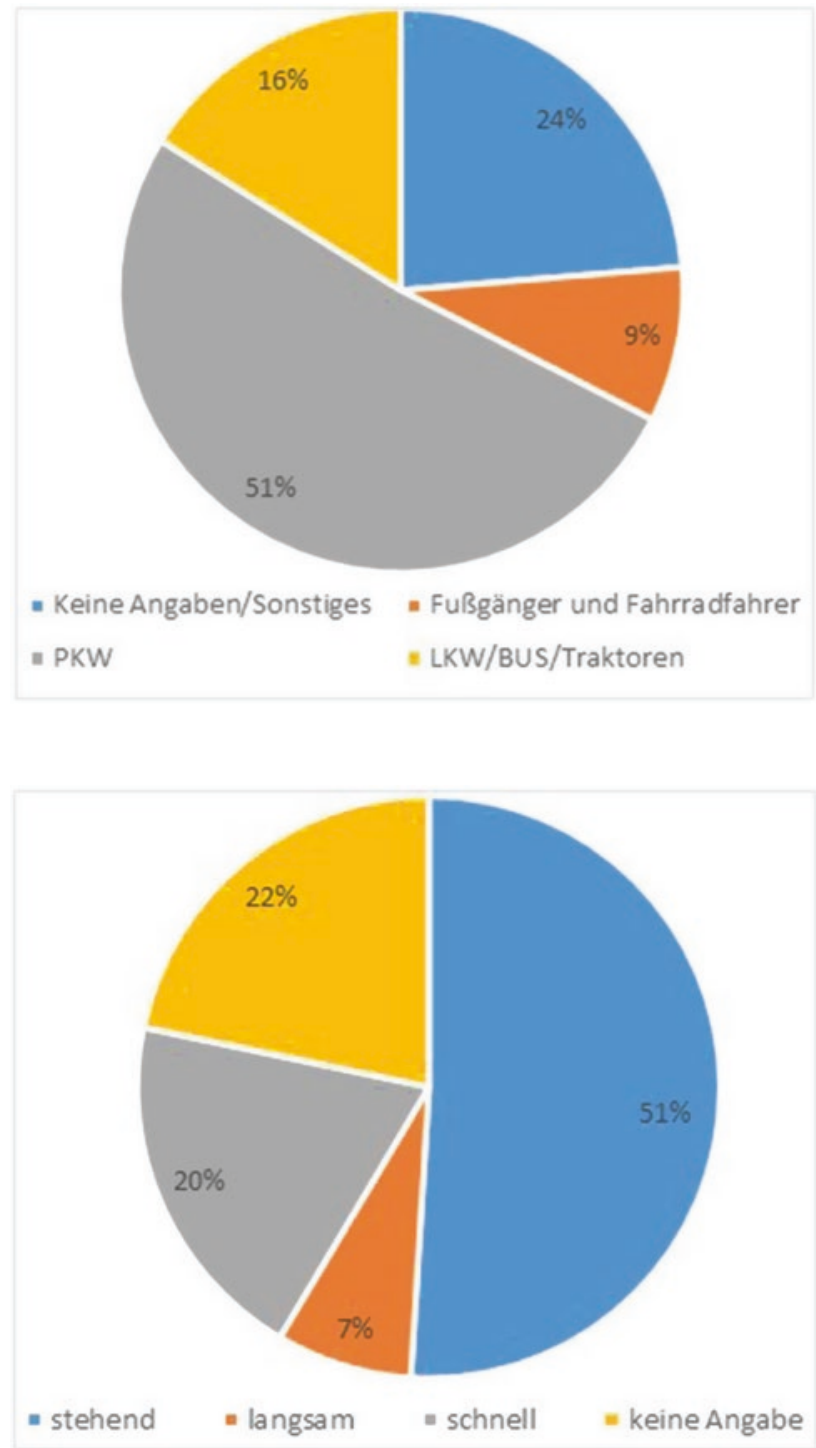

art dokumentiert. Abb. 5.11 zeigt die Ergebnisse der Unterteilung nach Hindernisart. Pkw mit einem Anteil von 51 \% sind hier als größter Störeinfluss auf den Bus zu nennen. Diese Störungen sind besonders häufig in den Bereichen A, AB, B, BC und C aufzufinden und bilden in diesen Bereichen einen Anteil von $78 \%$ aller Störungen durch Pkw. Betrachtet man parallel zu diesen Erkenntnissen Abb. 5.12, welche die Hindernisse entsprechend ihrer Geschwindigkeit unterteilt, fällt auf, dass der größte Anteil auf stehende Hindernisse (51\%) zurückzuführen ist. Falsch parkende Fahrzeuge an Bäckereien, Banken und Restaurants sind als Ursache für die häufigen Behinderungen des Busses zu sehen. Lieferverkehr, welcher an der Straßenseite oder in zweiter Reihe parkt, zählt ebenso zu dieser 
Gruppe der Störeinflüsse. Da der Bus in seiner aktuellen Konfiguration nicht selbstständig ausweichen bzw. seine Spur wechseln kann, müssen die Operatoren den Bus in solchen Situationen manuell steuern.

Weitere Hindernisse sind LKW und Busse, welche in den Bereichen C1, C2 und C3 ca. $16 \%$ aller Unterbrechungen auslösen. Diese Gruppe ist ebenfalls den stehenden Hindernissen aus Abb. 5.12 zuzuordnen. Fußgänger und Fahrradfahrer bilden mit $9 \%$ die kleinste Gruppe an Hindernissen und sind ebenfalls in den Bereichen C1, C2 und C3 (Fußgängerzone) anzutreffen, da es hier keine räumliche Trennung zwischen der Fahrbahn des autonomen Busses und dem Fußgängerweg gibt. Mit $20 \%$ bilden schnelle Fahrzeuge den zweitgrößten Anteil (Abb. 5.12), welche nur auf dem öffentlichen Straßenbereich mit Gegenverkehr (Bereich BC) dokumentiert wurden.

Werden Abb. 5.11 und 5.12 in Relation gestellt und man betrachtet nur die größte Hindernisart Pkw, stellt man fest, dass $62 \%$ aller Pkw stehend und $36 \%$ schnell fahrend zu einem Hindernis wurden.

Wertet man die Hindernishäufigkeit auf den einzelnen Streckenabschnitten aus, erhält man das Diagramm in Abb. 5.13. Besonderes Augenmerk ist hier auf die Bereiche AB und C2 zu legen. Hier werden in Summe ca. $50 \%$ aller Hindernisse gezählt. Die Ursache für diese Anhäufung ist die fehlende Trennung der einzelnen Verkehrsteilnehmer. Sowohl in der Innenstadt als auch in der Fußgängerzone erschweren Fußgänger, Lieferverkehr und

Abb.

5.13 Hindernishäufigkeit nach Streckenabschnitten (eigene Darstellung)

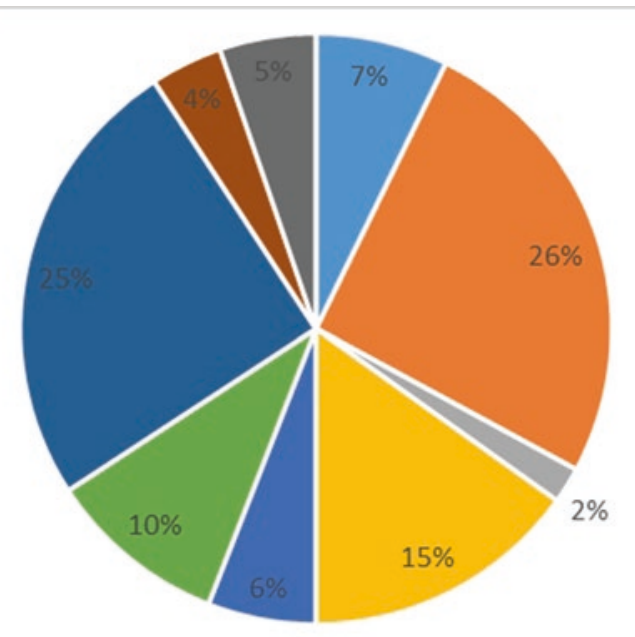

- Marktplatz (A)

- Innenstadtbereich (AB)

- Ampel/Ausfahrt zur Straße (B)

- Straße (BC)

- Fußgängerzone Einfahrt/Ausfahrt (C)

- Fußgängerzone bis Artrium (C1)

- Fußgängerzone Artrium bis Rottal Terme (C2)

- Überlandweg (C3)

- keine Angabe 
parkende Fahrzeuge den reibungslosen autonomen Fahrbetrieb des Busses. Häufig wird der Bus auch vorsätzlich an der Weiterfahrt gehindert, wenn z. B. Fahrbahnmarkierungen ignoriert werden. Ohne Operatoren, die manuell eingreifen können, wäre in diesen Bereichen ein reibungsloser Betrieb nicht möglich.

Wie bereits in Abschn. 5.3.2.2 erwähnt, verfügt der Bus über mehrere Möglichkeiten anzuhalten. Man unterscheidet einerseits das automatisch gesteuerte Verhalten des Busses mit Hilfe von Sensoren (d. h. Not-Stopp durch Bus: NB, Soft-Stopp durch Bus: SB) und andererseits das Verhalten der Operatoren (d. h. Not-Stopp durch Operator: NO, SoftStopp durch Operator SO), sowie die Komfortsituation. Eine Übersicht der Häufigkeitsverteilung der einzelnen Auslösungen zeigt Abb. 5.14. Man erkennt, dass bereits $76 \%$ aller Haltemanöver durch den Bus autonom erfolgen (NB und SB). Am häufigsten kam es im Bereich BC zu Not-Stopp-Situationen durch den Bus. Die häufigsten Soft-StoppSituationen des Busses wiederum gab es in den verkehrsreichen Gebieten AB und C2. Die Operatoren mussten in nur $16 \%$ aller Fälle mithilfe des manuellen Soft-Stopps den Bus abbremsen (ebenfalls in den Bereichen $\mathrm{AB}$ und $\mathrm{C} 2$ ). Ob der Bus in diesen von Operatoren gesteuerten Fällen nicht selbstständig angehalten hätte, bleibt allerdings offen. Die Auswertung hat gezeigt, dass einige Operatoren im Schnitt fünfmal häufiger den Soft-Stopp betätigen als ihre Kollegen. Einen Not-Stopp durch den Operator gab es in den dokumentierten Situationen nicht. Es sollte jedoch versucht werden, den Anteil der Not-Stopps durch den Bus auf ein Minimum zu reduzieren. Bei steigender Geschwindigkeit sind gerade die Not-Stopp-Situationen besonders gefährlich, da die Sitze nicht alle in Fahrtrichtung positioniert sind und der Bus schlagartig abgebremst wird.

Neben den Ankreuzmöglichkeiten des Dokumentationsbogens beinhaltete er ein weiteres Freifeld, in welches die Operatoren Besonderheiten eintragen konnten. Diese Beson-

Abb. 5.14 Abbremsverhalten des Busses (eigene

Darstellung)

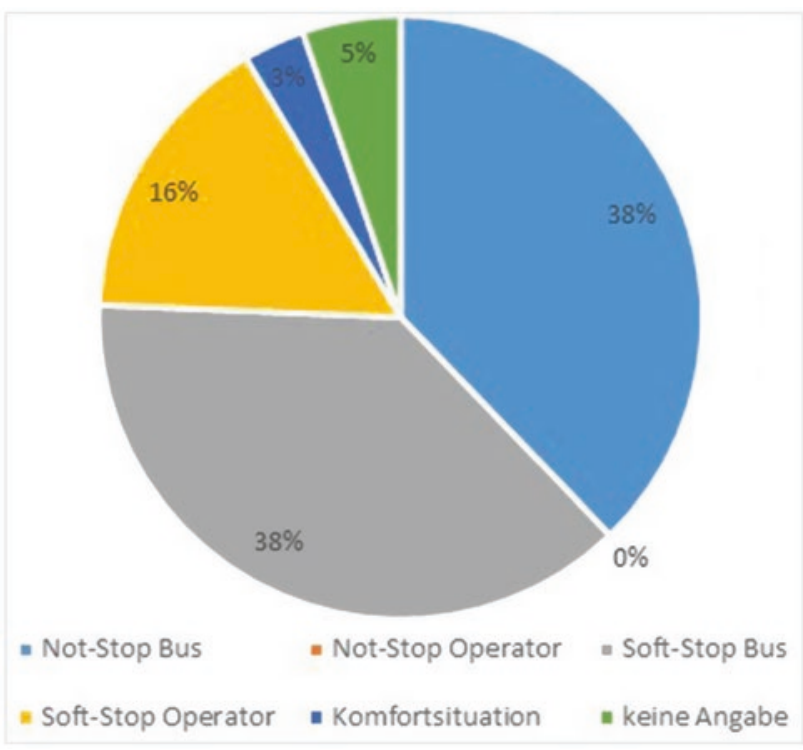


derheiten aus den Fragebögen und Interviews waren meist Einzelvorkommnisse, spielen aber eine entscheidende Rolle in der Entwicklung eines autonomen Fahrzeuges. Soll der Bus autonom fahren, also ohne das Eingreifen von Operatoren, müssen auch diese Spezialfälle durch die Technik des Fahrzeuges abgedeckt werden. Im Folgenden sind diese Besonderheiten und Einzelvorkommnisse zusammengefasst:

- Geöffneter Kanaldeckel hat den Busfahrer zu einem Soft-Stopp und zum manuellem Eingreifen veranlasst

- Bewässerungsanlage löst Not-Stopp durch den Bus aus

- Überholender Pkw schert kurz vor dem Bus ein und provoziert einen Not-Stopp

- Vom Wind bewegte lange Grashalme und Blätter zwingen den Bus in den Not-Stopp

- Kreuzende Katze löst automatischen Not-Stopp aus

- Aufsteigende Dämpfe aus Kanaldeckel und vom Außenbereich Rottal Terme führen zum Abbremsen des Busses

- Entgegenkommende Fahrzeuge mit überhöhter Geschwindigkeit im Bereich BC lösen Not-Stopp durch den Bus aus

- Seitlich zum Bus „schlendernde“ Fußgänger führen zu wiederholtem Abbremsen und Anfahren des Busses

- Unterschiedliche Reflektivität beim Einfahren in Bereich C führt zu Not-Stopp des Busses

Zusammenfassend kann gezeigt werden, dass der Bus trotz der umfangreichen Sensorik noch nicht gegen alltägliche Probleme gewappnet ist.

Als weiteres Problemfeld sind Systemfehler des Busses anzusehen, welche in allen Bereichen der Fahrstrecke auftraten. Bei dieser Art von Fehlern verliert der Bus ohne ersichtlichen Grund seine Lokalisierung und ist nicht in der Lage, autonom weiterzufahren. In diesen Situationen müssen die Operatoren den Bus manuell bewegen und das System neu starten. $22 \%$ aller dokumentierten Situationen waren mit einem Systemfehler verbunden, von denen $87 \%$ ohne Hindernis auftraten.

Damit der Bus zukünftig alle Situationen autonom bewältigen kann, muss die Sensorik optimiert werden. Durch Versuche mit dem Shuttlebus im Fahrbetrieb sollen bisher nicht aufgetretene Problemfälle aufgedeckt werden. Hierfür wurden im weiteren Verlauf des Projektes Versuchskonfigurationen definiert.

\subsubsection{Phase 2.2 - Versuchskonfigurationen}

Wie bei der Erstellung und Auswertung der Dokumentationsbögen wurden die Versuchskonfigurationen in Einzelbereiche untergliedert, um eine systematische Durchführung der Tests zu ermöglichen. Hierfür wurden drei Teilbereiche mit den entsprechenden Konfigurationen gebildet:

Bereich 1: Überprüfung und Ermittlung von Herstellerangaben bezüglich der Sensorfunktionen 
In diesem Bereich sollen die Angaben von Sicherheitsabständen für den Not- bzw. SoftStopp des Busses evaluiert, Angaben und Einstellungen der Sensoren überprüft sowie Einbauhöhe und Öffnungswinkel verifiziert werden. Durch Versuche mit Hindernissen, die größer bzw. kleiner als $30 \mathrm{~cm}$ sind, soll die Notwendigkeit einer Sensorfusion deutlich gemacht werden.

Bereich 2: Überprüfung von Operatorenaussagen

Die größte Erfahrung mit auftretenden Problemen während des Betriebes haben die Operatoren bei ihren täglichen Fahrten gesammelt, in den Dokumentationsbögen niedergeschrieben und in Interviews geschildert. Diese Probleme sollen in der Versuchsdurchführung möglichst äquivalent nachgestellt und Ursachen für beschriebene Fehler definiert werden. Dazu zählen die bereits im Abschn. 5.3.2.3 erwähnten unterschiedlichen Verhaltensweisen des Busses bei starker Reflektion von Leitzylindern im Bereich C, parkenden Fahrzeugen am Straßenrand in unterschiedlichen Abständen und im Straßenbereich BC bei zu schnell entgegenkommenden Fahrzeugen. Weiterhin sollen Einflüsse der Umwelt wie Regen und Nebel nachgestellt werden. Aufgespannte $(>30 \mathrm{~cm}) \mathrm{bzw}$. aufgestellte $(<30 \mathrm{~cm})$ Hindernisse sollen die realen Gegebenheiten nachstellen. Fahrzeuge sollen mit einem Fahrzeugtarget (4activesystems 2017) simuliert werden. Um Fahrzeugbewegungen (z. B. Gegenverkehr) zu simulieren, soll eine überfahrbare Roboter Plattform (DSD o. J.) verwendet werden.

Bereich 3: Nachstellung von Spezialfällen und besonderen Vorkommnissen

Spezielle Vorkommnisse, welche nur vereinzelt aufgetreten sind, scheinen aufgrund der geringen Häufigkeit nicht relevant. Dennoch muss auf diese Fälle besonderes Augenmerk gelegt werden, um unfallfreies autonomes Fahren zu ermöglichen. Zu diesen vereinzelten Vorkommnissen zählen Hindernisse (siehe Abschn. 5.3.2.3), welche jahreszeitbedingt auftreten. Verwirbeltes Laub im Herbst oder hohes Gras im Frühling sind als solche temporären Hindernisse einzuordnen und sollen in der Versuchsdurchführung nachgestellt und evaluiert werden. Die Auswertung der Dokumentationsbögen hat außerdem gezeigt, dass Spezialfälle, wie ein seitlich zum Bus schlendernder Fußgänger oder eine kreuzende Katze, den Bus an seine technischen Grenzen bringen und zu unkomfortablen Situationen führen. Diese speziellen, alltäglichen Gegebenheiten gilt es bestmöglich (z. B. ein Ball simuliert eine Katze) nachzustellen.

\subsubsection{Phase 2.3 - Versuchsdurchführung}

Alle Versuche wurden mit dem Bus Gen1 2.0 durchgeführt. Der laufende Betrieb an den Versuchstagen wurde für die Versuchsdurchführung zeitweise unterbrochen. Um die Unterbrechungen möglichst kurz zu halten, stand der Bus inklusive Operator zeitlich begrenzt zur Verfügung. 
Die angegebenen Abstände zur Busfahrspur beziehen sich auf die Außenkante des Vorderrades. Die Herstellerangaben hinsichtlich der Sicherheitsabstände beziehen sich auf die Verkleidung der Sensorik, die $22 \mathrm{~cm}$ weiter nach außen ragt. Dieser Abstand wird in der Auswertung von den gemessenen Werten abgezogen.

In der ersten Versuchsreihe wurde geprüft, wie sensibel das System auf Hindernisse, die direkt im Fahrschlauch des Busses platziert sind, reagiert. Aufgrund der großzügigen Platzverhältnisse wurde Abschnitt C3 zum Durchführen der Tests gewählt. Die Testobjekte wurden in einem ausreichenden Abstand zur Startposition des Busses in der Mitte der Fahrbahn platziert, sodass der Bus seine Geschwindigkeit von $12 \mathrm{~km} / \mathrm{h}$, wie für diesen Streckenabschnitt vorgesehen, bei jedem Versuch erreichen konnte. Dadurch wurde sichergestellt, dass alle Versuche reproduzierbar durchgeführt werden konnten. Nach jedem Test wurde der Bus durch den Operator manuell zurück zur Startposition gefahren. Um den nächsten Versuch zu starten, leitete der Busoperator den autonomen Fahrbetrieb ein.

Begonnen wurde mit Holzelementen, deren Höhe schrittweise vergrößert wurde. Der Bus überfuhr ohne Reaktion das Holzelement mit einer Höhe von 1,6 cm und einer Breite von $150 \mathrm{~cm}$ (Versuch 1.1). Im zweiten Versuch wurde das Holzelement aufgestellt, sodass das Hindernis eine Höhe von $15 \mathrm{~cm}$ und eine Breite von $150 \mathrm{~cm}$ hatte. Der Bus überrollte ohne Reaktion das Brett, das durch den Reifenkontakt umgestoßen wurde, und führte die Fahrt wie gewohnt weiter (Versuch 1.2). Mehrere, übereinander platzierte Holzelemente mit einer Höhe von $18 \mathrm{~cm}$ wurden durch den Bus nicht erkannt. Erst als die Holzelemente von den Rädern erfasst wurden, kam es durch die Erschütterungen zu einem automatischen Not-Stopp (Versuch 1.6). Bei einer Hindernishöhe von $27 \mathrm{~cm}$ hielt der Bus kurz an, wartete einige Sekunden und beschleunigte anschließend wieder, sodass es zu einer Kollision mit dem Hindernis kam. Es wird vermutet, dass die Holzelemente kurzzeitig in den Sichtbereich der Umfeldsensorik gerieten, was auf Bodenunebenheiten und Fahrwerksbewegungen des Busses zurückzuführen sein könnte (Versuch 1.15). Bei der Wiederholung des Versuchs erkannte der Bus das Hindernis nicht und kollidierte ohne vorheriges Anhalten mit den aufgestellten Elementen (Versuch 1.16). Die Höhe des Hindernisses wurde um weitere 1,6 cm auf 28,6 cm erhöht. Es wurde jedoch keine Änderung im Verhalten des Busses festgestellt. Nach einem Soft-Stopp beschleunigte der Bus wieder automatisch und kollidierte mit dem Hindernis (Versuch 1.17). Zwei übereinander platzierte Holzelemente mit einer Gesamthöhe von $30 \mathrm{~cm}$ führten zu einem Soft-Stopp des Busses $2 \mathrm{~m}$ vor dem Hindernis (Versuch 1.3). Das gleiche Ergebnis ergab der Versuch mit einem $40 \mathrm{~cm}$ hohen, schwarzen Hindernis (Versuch 1.19). Zum Schluss der ersten Versuchsreihe wurden zwei Extremfälle nachgebildet. Im ersten Fall wurde ein Fußgängerdummy in der Fahrspur des Busses liegend platziert. Der Dummy wurde 2016 an der Technischen Hochschule Ingolstadt entwickelt. Seine Abmessungen repräsentieren die eines durchschnittlichen Erwachsenen (Doric 2017). Durch die geringe Höhe des Dummys konnte der Bus das Objekt nicht detektieren und folglich wurde kein automatischer Soft-Stopp eingeleitet. Zur Vermeidung einer Kollision musste der Operator den Not-Stopp manuell einleiten (Abb. 5.15, Versuch 1.21). 


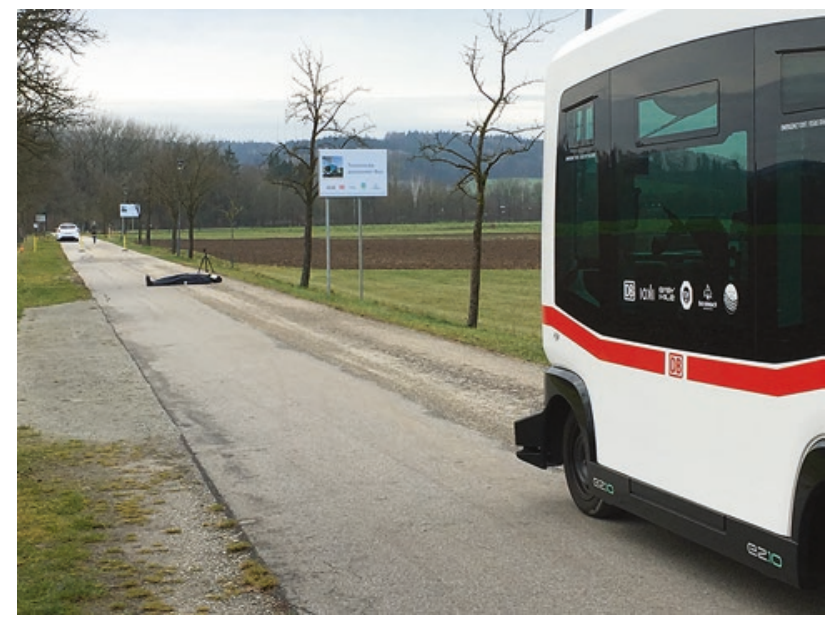

Abb. 5.15 Versuch 1.21 (eigene Darstellung)

Im zweiten Extremfall wurde ein weißes Tuch auf dem Boden platziert, welches ein stark reflektierendes, flaches Hindernis darstellt. Das Objekt wurde auch in diesem Versuch vom Bus ohne Reaktion überrollt (Versuch 1.20).

In der zweiten Versuchsreihe wurde der Einfluss des seitlichen Abstands eines Hindernisses zum vorbeifahrenden Bus untersucht. Gleichzeitig wurden die Auswirkungen des Reflexionsverhaltens betrachtet. In den einzelnen Versuchen wurde die Pylone teilweise mit Reflektor getestet, deren Reflektor auf Sensorhöhe ausgerichtet wurde. Zum Vergleich wurde eine Pylone verwendet, deren Reflektor mit schwarzem Tape überklebt wurde. Bei 0,9 m seitlichem Abstand der stark reflektierenden Pylone zur Fahrspur des Busses verzögerte der Bus auf 2 km/h und nahm im Nachgang wieder Fahrt auf (Abb. 5.16, Versuch 2.6). Die abgeklebte, schwächer reflektierende Pylone rief ein Abbremsen auf nur $9 \mathrm{~km} / \mathrm{h}$ hervor (Versuch 2.7). Bei einem Abstand von 0,7 m verzögerte der Bus an der unveränderten Pylone auf weniger als $1 \mathrm{~km} / \mathrm{h}$ und benötigte einige Zeit, bis die Vorbeifahrt beendet war. Anschließend beschleunigte der Bus auf die vorgegebene Geschwindigkeit (Versuch 2.10). Bei der schwächer reflektierenden Pylone bremste der Bus lediglich auf $6 \mathrm{~km} / \mathrm{h}$ ab (Versuch 2.11). Eine weitere Verringerung des Abstandes auf 0,6 m führte bei dem reflektierenden Objekt zu einem kurzen Anhalten, gefolgt von herantastenden Anfahrversuchen. Zum Schluss blieb der Bus mittels Soft-Stopp stehen (Versuch 2.14). Die verklebte Pylone führte von anfänglichen Brems- und Beschleunigungsvorgängen zu einem automatischen Not-Stopp (Versuch 2.15). Verkürzt man den seitlichen Abstand erneut, auf diesmal 0,5 m, löste der Bus bei der reflektierenden Pylone einen Soft-Stopp in einem Abstand von $2 \mathrm{~m}$ aus (Versuch 2.12). Bei der abgeklebten Pylone tastete sich der Bus bis auf $45 \mathrm{~cm}$ heran, um dann einen Not-Stopp auszulösen (Versuch 2.13).

In einer weiteren Versuchsreihe wurde die Reaktion des Busses auf Pylonen mit größerem seitlichen Abstand untersucht. Bei einem seitlichen Abstand von $160 \mathrm{~cm}$ zwischen der Busfahrspur und der stark reflektierenden Pylone bremste der Bus von $12 \mathrm{~km} / \mathrm{h}$ auf $9 \mathrm{~km} / \mathrm{h}$ 
Abb. 5.16 Versuch 2.6 (eigene Darstellung)

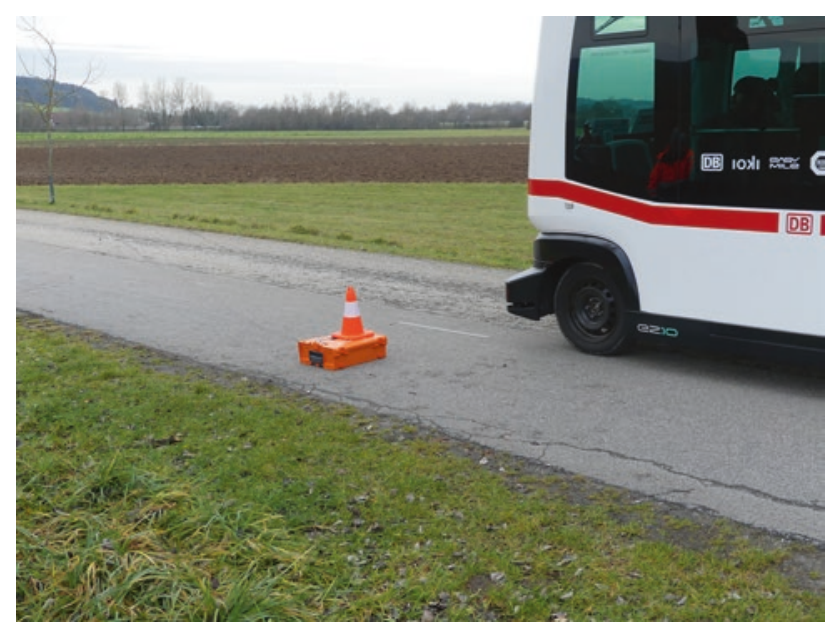

ab und passierte das seitlich stehende Hindernis (Versuch 8.1). Bei der abgeklebten Pylone bremste der Bus lediglich auf $11 \mathrm{~km} / \mathrm{h}$ ab (Versuch 8.2). Eine Vergrößerung des seitlichen Abstandes auf $170 \mathrm{~cm}$ führte bei der reflektierenden Pylone zu einer Verringerung der Geschwindigkeit auf $11 \mathrm{~km} / \mathrm{h}$ beim Passieren (Versuch 8.4). Bei der abgeklebten Pylone zeigte der Bus keine Reaktion auf das Hindernis (Versuch 8.3). Der Abstand von $180 \mathrm{~cm}$ führte bei keiner der Pylonen zu einem Abbremsvorgang des Busses (Versuch 8.5, Versuch 8.6).

Das 4activeC2-Fahrzeugtarget wurde innerhalb der nächsten Versuchsreihe in verschiedenen seitlichen Abständen zur Fahrspur des Busses platziert. An der getesteten Stelle erreichte der Bus ohne Hindernis eine Endgeschwindigkeit von $9 \mathrm{~km} / \mathrm{h}$. Die seitliche AuBenkante des Fahrzeugtargets wurde im ersten Versuch in einem Abstand von $20 \mathrm{~cm}$ parallel zur Busfahrspur platziert. Der Bus hielt $2 \mathrm{~m}$ vor dem Target mittels Soft-Stopp an (Versuch 6.1). Das gleiche Verhalten zeigte sich bei Abständen von $30 \mathrm{~cm}$ und $40 \mathrm{~cm}$ (Versuch 6.2, Versuch 6.3). Bei $50 \mathrm{~cm}$ Abstand zur Fahrspur kam ein zögerliches Verhalten des Busses zustande, das während des Herantastens in einem Soft-Stopp in der Nähe des Targets endete (Versuch 6.4). Beim Platzieren des Targets mit einem Abstand von $60 \mathrm{~cm}$ tastete sich der Bus ebenfalls heran und passierte das Target mit einer Geschwindigkeit von $1 \mathrm{~km} / \mathrm{h}$ (Versuch 6.5).

In der darauffolgenden Versuchsreihe wurde das 4activeC2-Fahrzeugtarget an einer Stelle platziert, an welcher der Bus ohne Hindernis $12 \mathrm{~km} / \mathrm{h}$ erreicht. Bei $160 \mathrm{~cm}$ Abstand der Target-Außenkante zur Busfahrspur bremste der Bus auf $9 \mathrm{~km} / \mathrm{h}$ ab und passierte das Target (Versuch 7.5). Bei einem eingestellten Abstand von $170 \mathrm{~cm}$ verlangsamte der Bus auf $11 \mathrm{~km} / \mathrm{h}$ (Versuch 7.7). Die $180 \mathrm{~cm}$ Abstand führten dazu, dass der Bus ohne bemerkbare Reaktion mit $12 \mathrm{~km} / \mathrm{h}$ am Target vorbeifuhr (Abb. 5.17, Versuch 7.6).

Die nächste Versuchsreihe beinhaltete Hindernisse, die nicht direkt auf dem Boden stehen. Sie wurden mittels einer Schnur, die über die Fahrbahn gespannt wurde, befestigt. Damit befanden sich die Elemente in $115 \mathrm{~cm}$ Höhe schwebend über der Fahrspur des Busses. Sowohl die schwebende Pylone als auch das in einem separaten Versuch 


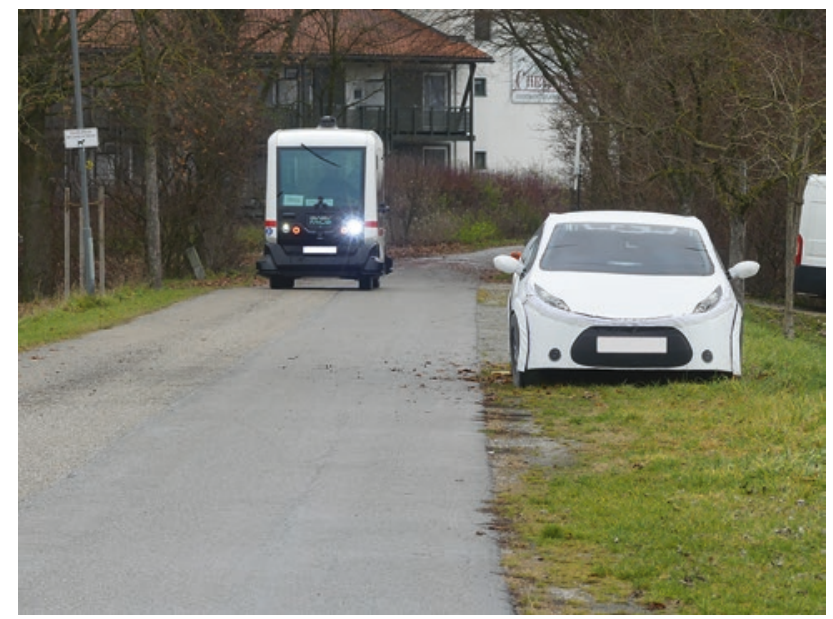

Abb. 5.17 Versuch 7.6 (eigene Darstellung)

aufgehängte weiße Tuch wurden durch den Bus nicht erkannt, sodass es zu einer Kollision kam (Versuch 3.1, Versuch 3.2).

Die Umwelteinflüsse konnten ebenfalls validiert werden. Am ersten Testtag konnte der reguläre Busbetrieb erst verspätet starten, da Nebel keine autonome Fahrt zuließ und der Betrieb währenddessen laut Zulassungsunterlagen untersagt ist.

Als der reguläre Fahrbetrieb möglich war, wurde Laub vor den fahrenden Bus geblasen. Nach zögerlicher Bremsung auf $8 \mathrm{~km} / \mathrm{h}$ folgte ein automatischer Not-Stopp des Busses (Versuch 4.1). In einem weiteren Versuch wurde der rechte, vordere Lidarsensor vor Fahrtantritt mit Wasser besprüht. Die Benetzung mit Wasser führte dazu, dass sich der Bus nicht in den autonomen Fahrbetrieb überführen ließ (Versuch 12.1).

In einem neuen Versuch wurde ein Schild, das der Bus für die Lokalisierung benötigt, mit einem schwarzen Tuch verdeckt. Der Bus zeigte keine Reaktion und passierte das Schild anstandslos (Versuch 9.1).

Der Einfluss dynamischer Targets wurde in den nachfolgenden Versuchsreihen untersucht.

Ein elastischer Kunststoffball wurde seitlich in Richtung des fahrenden Busses gerollt. Der Ball näherte sich in den Versuchen schnell dem Bus an und berührte ihn jeweils seitlich vorne, seitlich mittig und seitlich hinten. Bei allen Versuchen erfolgte ein automatischer Not-Stopp (Versuch 10.1, Versuch 10.2, Versuch 10.3). Bei einem weiteren Versuch wurde der Ball von hinten an die Rückseite des Busses gerollt. Hierbei zeigte der Bus keine Reaktion (Versuch 10.5).

Das 4activeC2-Target wurde auf einer überfahrbaren Roboterplattform befestigt. Im ersten Versuch fuhr die Plattform vor dem Bus her und bremste an verschiedenen Stellen. Der Bus näherte sich mit $12 \mathrm{~km} / \mathrm{h}$ an und verringerte die Geschwindigkeit bei Bedarf, damit ein Mindestabstand von $2 \mathrm{~m}$ eingehalten wurde (Versuch 5.1). Als die Plattform bis zum Stillstand bremste und rückwärts auf den Bus zufuhr, löste der Bus einen Not-Stopp 
aus (Versuch 5.4). Im folgenden Versuch wurde das Verhalten eines sich von hinten annähernden Fahrzeuges nachgestellt. Das Fahrzeugtarget fuhr bis auf wenige Zentimeter auf den Bus auf, ohne dass der Bus eine Reaktion zeigte (Versuch 5.6).

In den darauffolgenden Versuchen sollten Situationen mit Gegenverkehr nachgestellt werden. Hierfür wurden wieder die Plattform und das 4activeC2-Target eingesetzt. Als Versuchsstrecke diente der Abschnitt C1. Der Bus bewegte sich an der getesteten Stelle mit 9 km/h ohne Gegenverkehr. Im Abstand von $65 \mathrm{~cm}$ zum Sensor fuhr die Plattform inklusive Fahrzeugtarget mit jeweils 5 km/h, 10 km/h, 15 km/h entgegen der Busfahrrichtung. Bei allen drei Geschwindigkeiten verlangsamte der Bus seine Fahrt auf $3 \mathrm{~km} / \mathrm{h}$. Mit steigender Target-Geschwindigkeit erfolgte der Bremsvorgang spürbar schneller (Versuch 13.2, Versuch 13.3, Versuch 13.4).

In der letzten Versuchsreihe wurde der Einfluss des Verhaltens von Fußgängern auf den Fahrbetrieb untersucht. Eine Versuchsperson lief beim ersten Versuch längs der Straße mittig auf der Busfahrspur vor dem Bus mit variierenden Geschwindigkeiten. Der Bus hielt einen Mindestabstand von $2 \mathrm{~m}$ ein. Als der Fußgänger auf der Fahrbahn stehen blieb, hielt der Bus ebenfalls an (Versuch 11.1). Beim zweiten Versuch lief die Versuchsperson links neben der Busfahrspur mit einem Abstand von über $1 \mathrm{~m}$ längs der Fahrtrichtung. Der Bus wurde beim Annähern an den Fußgänger langsamer. Als der Fußgänger stehen blieb, passierte der Bus diesen mit einer Geschwindigkeit von 1 km/h (Versuch 12.2). Bei einer weiteren Versuchskonfiguration bewegte sich der Fußgänger erneut längs zur Busfahrspur und schlenderte dabei in Querrichtung. Dadurch änderte sich der Abstand zwischen Fußgänger und Bus fortlaufend. Der Bus variierte die Geschwindigkeit je nach Abstand des Fußgängers. Als sich der Fußgänger, der weiter vorne als der Bus war, schnell in die Fahrspur des Busses bewegte, kam bei zu geringem Abstand ein Not-Stopp zu Stande. Als der Bus am Fußgänger langsam vorbeifuhr und der Fußgänger sich dem Seitenteil des Busses bis auf Körperkontakt näherte, wurde in der Regel ein Not-Stopp ausgelöst. Lediglich ein Versuch ergab ein anderes Ergebnis (Versuch 11.3). Es konnte aber nicht geklärt werden, worin die Ursache für dieses Verhalten lag.

\subsubsection{Phase 3 - Versuchsanalyse}

An drei aufeinanderfolgenden Testtagen wurden 84 Einzelversuche durchgeführt. Die Versuchsanordnungen entsprechen den in Abschn. 5.3.2.4 festgelegten Versuchskonfigurationen. Die zu Beginn der Versuche durchgeführten Referenzmessungen auf der Teststrecke ohne Hindernisse ergeben, dass die gewählte Teststrecke keinen Einfluss auf die Versuchsergebnisse hat. Die Überprüfung der Sensorfunktionen durch Abdeckung der Sensoren zeigte, dass die Wirksamkeit selbiger den Herstellerangaben für den Bus Gen1 2.0 entspricht.

Die Überprüfung der Herstellerangaben der seitlich geltenden Sicherheitsabstände sowie die Ermittlung des Perimeters d1 an der Front des Busses zeigen, dass unbewegliche Objekte, die auf dem Boden stehen und eine Ausdehnung in der Höhe von $>30 \mathrm{~cm}$ haben, zuverlässig erkannt werden. Die Geschwindigkeitsreduktion bei stehenden Objekten im Frontbereich sowie seitlich zum Bus im Abstand zwischen Perimeter d1 und d2 erfolgt zuverlässig. Beim Erreichen des Perimeters d1 stoppt der Bus wie vorgegeben. 
Bei Objekten vor dem Shuttlebus verringert dieser den Abstand mit abnehmender Geschwindigkeit bis auf einen Abstand von 2 m, wo ein Soft-Stopp ausgelöst wird. Der Wert von $2 \mathrm{~m}$ wurde in mehreren Versuchen mit unterschiedlichen Hindernissen ermittelt und entspricht somit dem Perimeter d1 im Frontbereich. Der geschwindigkeitsabhängige Perimeter d2 konnte innerhalb der durchgeführten Versuchsreihen nicht ermittelt werden. Wie die Auswertung der Dokumentationsbögen vermuten lässt, zeigen die Versuche, dass auf gängige Objekte im Fahrschlauch mit ausreichender Sicherheit reagiert und ein Unfall verhindert wird.

Die Versuche mit stehenden Objekten im Seitenbereich des Shuttles bestätigen ebenfalls die Herstellerangaben und die Zuverlässigkeit der Sicherheitsvorschriften. Sowohl aufgestellte Pylonen als auch Fahrzeuge werden zuverlässig erkannt und reduzieren die Geschwindigkeit des Busses, sobald diese mit einem Abstand von $<150 \mathrm{~cm}$ zum Bus stehen. Objekte mit einer Positionierung $<30 \mathrm{~cm}$ seitlich zum Shuttle lösen einen Soft-Stopp aus.

Die Versuche mit bewegten Objekten bestätigten ebenfalls die angegebenen Abstände und deren Richtigkeit für den zugelassenen Geschwindigkeitsbereich. Sie zeigen zusätzlich, dass die Funktion des Soft-Stopps funktioniert und gut umgesetzt ist, da der Bus bei den entsprechenden Voraussetzungen eigenständig wieder anfährt. Ebenfalls wird die Funktion des Not-Stopps validiert.

Fahrzeuge und Fußgänger, die sich vor dem Shuttlebus bewegen, rufen die festgelegten Reaktionen hervor. Ab Unterschreitung des Perimeters d2, dessen Wert nicht ermittelt werden konnte, verringert der Shuttlebus seine Geschwindigkeit. Sobald der Abstand von $2 \mathrm{~m}$ erreicht wird, löst der Bus einen Soft-Stopp aus. Vergrößert sich der Abstand durch die Bewegung des Fahrzeuges oder Fußgängers wieder, setzt der Bus seine Fahrt fort.

Versuche im Heckbereich des Busses zeigen, dass es dort, wie angegeben, keinen Sicherheitsabstand gibt. Bei den Versuchen mit dem Fahrzeug-Target, das mittels Plattform auf den Bus auffährt, erfolgt bis zu dem getesteten Abstand von $1 \mathrm{~m}$ keine Reaktion. Selbst bei der Unterschreitung des Abstandes bis hin zum Kontakt, hervorgerufen durch einen Ball, ist keine Reaktion erkennbar. Die Reaktion entspricht dem Verhalten eines ,echten“ Fahrers der einen drohenden Auffahrunfall nicht erkennt.

Die Überprüfung der seitlichen Sicherheitszonen zeigt, dass die Perimeter d1 (= $30 \mathrm{~cm})$ und d2 (= $150 \mathrm{~cm})$ korrekt angegeben sind. Beim Passieren eines Fußgängers, der sich seitlich zum Fahrzeug bewegt, reduziert der Shuttlebus zuverlässig die Geschwindigkeit, sofern der Abstand zwischen den Perimetern d2 und d1 liegt. Schwankt der Abstand, reagiert der Bus entsprechend. Der ständige Geschwindigkeitswechsel wird nach eigenem Empfinden und Aussagen von Passagieren allerdings als störend empfunden. Nach Passieren des Fußgängers wird wieder auf die vorgegebene Geschwindigkeit beschleunigt. Gleiches gilt für entgegenkommende Fahrzeuge, selbst wenn diese mit unterschiedlichen Geschwindigkeiten vorbeifahren. Sollte der Abstand den Perimeter d1 unterschreiten, in den Versuchen durch einen Fußgänger, der an den Bus springt, abgebildet, vollzieht der Bus einen Not-Stopp.

Die Überprüfung der Herstellerangaben zeigt allerdings einige Schwachstellen auf. Bei Objekten, die sich von vorne auf das Fahrzeug zubewegen, reagiert der Bus wie beabsich- 
tigt. Bei einem Abstand von 2 m wird die Fahrt gestoppt. Die weitere Verringerung des Abstandes, also das Unterschreiten des Perimeters d1, durch die Bewegung des gegnerischen Objektes, löst einen Not-Stopp aus. Sofern das nahende Objekt seine Bewegung nicht unterbricht, kommt es zur Kollision. Die Parallele zu dem einzigen Unfall des Shuttles, der bis Dezember 2018 erfolgte, bei dem ein ausparkendes Fahrzeug vorne an den Bus fuhr, ist hier deutlich zu erkennen (Passauer Neue Presse 2018). Zu einem Kontakt mit dem Bus kommt es ebenfalls bei Objekten, die sich von der Seite oder von hinten dem Bus nähern. Der klassische Seitenaufprall und Auffahrunfall kann, ähnlich wie bei Fahrzeugen mit „echten“ Fahrern, nicht verhindert werden. Dass dies für den Einsatz des Busses kein K.O.-Kriterium darstellt, könnte an der Schuldfrage liegen. Schuld hätte in diesem Fall der Unfallgegner und nicht der Shuttlebus.

Sicherheitslücken, bei denen es zu einem Unfall mit Verschulden des Busses kommt, können bei den Versuchen mit stehenden Objekten, mit einer Höhe von unter $30 \mathrm{~cm}$, und auf über $30 \mathrm{~cm}$,,schwebenden“ Objekten festgestellt werden.

Objekte, die deutlich kleiner als $30 \mathrm{~cm}$ sind und sich im Fahrschlauch des Shuttlebusses befinden, werden durch den einstrahligen Lidarsensor nicht erkannt und lösen somit keine Reaktion aus. Im besten Fall wird dadurch lediglich der Bus beschädigt. Wobei es vorstellbar ist, dass sich die Insassen durch die ruckartige Bewegung des Busses verletzen. Der Versuch mit dem auf der Straße liegenden Dummy zeigt aber, dass es bei dieser Sicherheitslücke auch außerhalb des Busses zu Personenschäden kommen kann.

Bei einem Objekt, das sich über dem Abtastbereich des einstrahligen Sensors und dessen „Halterung“ sich außerhalb der Sicherheitsabstände befindet, kommt es zum Unfall. Dies wäre der Fall, wenn eine Ladung nach hinten über das Fahrzeugende hinaussteht (Abb. 5.18). Laut Straßenverkehrsordnung (BfJ 2013) darf der Überstand bis zu $3 \mathrm{~m}$ lang

Abb. 5.18 Beispiel für überstehende Ladung (eigene Darstellung)

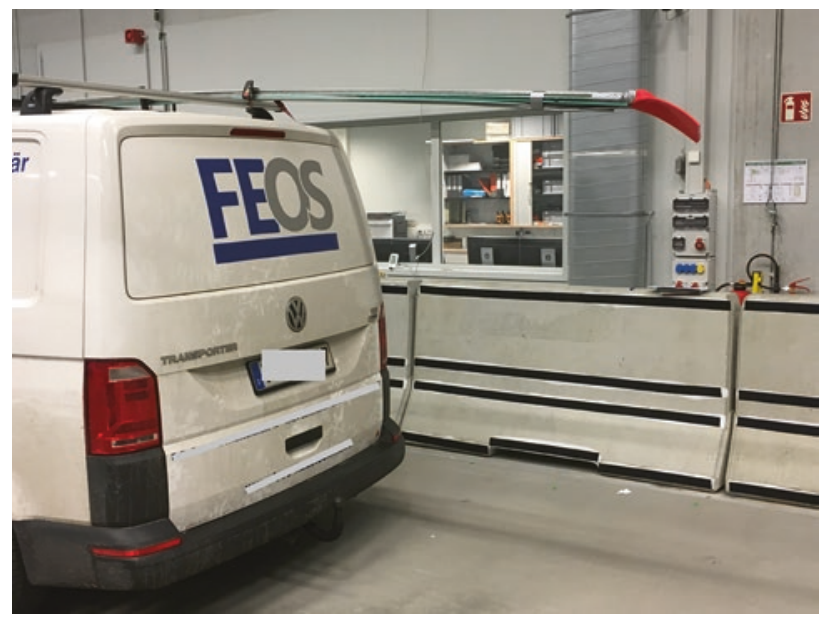


sein. Der Abstand des Perimeters d2 (= $2 \mathrm{~m})$ würde damit nicht ausreichen, um vor dem Ende der Ladung zu stoppen.

Gleiches gilt für Fahrzeuge, bei denen der Fahrzeugboden mehr als $30 \mathrm{~cm}$ über der Straße liegt und sich die Räder mehr als 2 m vor dem Fahrzeugende befinden. Deutlich wird dies anhand eines Reisebusses mit rückseitig angebrachter Transportkiste (Abb. 5.19).

Aus den Ergebnissen der durchgeführten Versuche lässt sich schlussfolgern, dass die über die Dokumentationsbögen erfasste Situation eines offenen Kanaldeckels ebenso wenig durch die Sensoren des Busses erfasst wird.

Die aufgezeigten Sicherheitslücken der Technik des Shuttlebusses Gen1 2.0 bedingen, dass ein Operator an Bord für den unfallfreien Betrieb notwendig ist. Es ist davon auszugehen, dass bei dem Betrieb des Busses der Gen1 2.3, bei dem ein zusätzlicher MultiLidarsensor im Front- und Heckbereich zum Einsatz kommt, die meisten der aufgezeigten Sicherheitslücken geschlossen sind und der Shuttlebus auch ohne Eingreifen des Operators keinen Unfall verursacht.

Neben den Herstellerangaben werden dokumentierte Einzelfälle und Einschätzungen der Operatoren überprüft. Sofern möglich, wurden diese mittels Versuchen nachgestellt. Teilweise mussten diese den örtlichen Versuchsmöglichkeiten angepasst werden.

Eine der Aussagen besagt, dass die Geschwindigkeit des entgegenkommenden Fahrzeuges Ursache für einen Not-Stopp war. Bei den durchgeführten Versuchen, die lediglich mit einer Geschwindigkeit bis 15 km/h erfolgten, konnte kein Einfluss der Geschwindigkeit des Gegenverkehrs nachgewiesen werden.

Der Einfluss der Umwelt wird hingegen deutlich. Die Versuche mit Wasser auf der Sensorabdeckung zeigen deutlich, dass Regen oder, wie dokumentiert, Wasser von Beregnungsanlagen die Fahrt unterbrechen oder das Anfahren des Busses verhindern. Hierdurch entsteht keine Reduzierung der Sicherheit, da das Stoppen des Shuttlebusses nicht

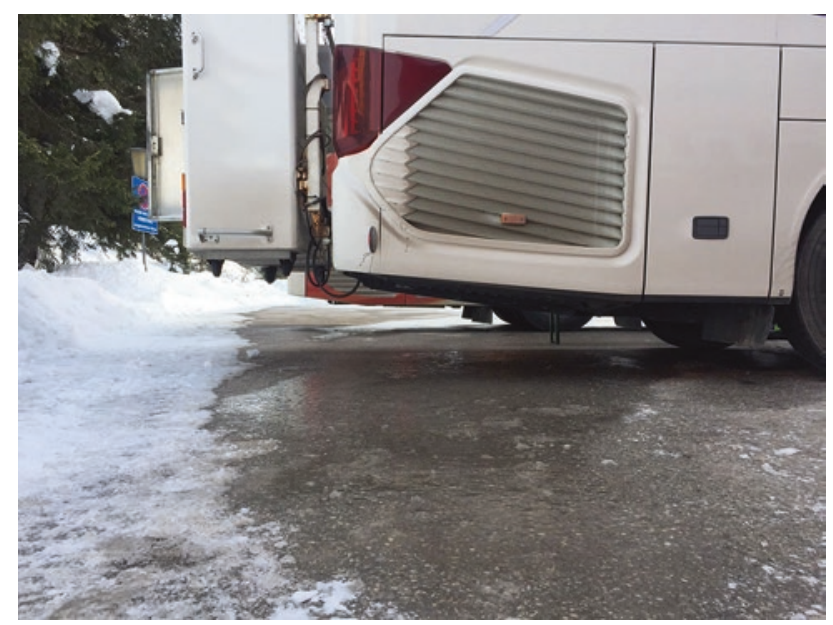

Abb. 5.19 Reisebus mit Transportkiste (eigene Darstellung) 
zwangsläufig eine Gefahrensituation auslöst. Allerdings wird deutlich, dass hierdurch kein durchgängiger und geregelter Betrieb im ÖPNV gewährleistet ist. Es ist davon auszugehen, dass eine andere Einstellung der Sensibilität der Sensorik leichten Regen und Verschmutzungen beherrschen könnte. Allerdings würde dies die gesamte Objekterkennung reduzieren, was wiederum zu einer Erhöhung der Unfallwahrscheinlichkeit führen würde. Daher ist die Vorgabe der Zulassungsauflagen, den Busbetrieb bei Regen und Schnee einzustellen, nachvollziehbar.

$\mathrm{Zu}$ den dokumentierten Auswirkungen von Nebel oder aus der Kanalisation aufsteigendem Dampf konnten keine Versuche durchgeführt werden. Bijelic et al. (2018) und Hasirlioglu und Riener (2017) zeigen allerdings auf, dass Nebel den Sichtbereich von Lidarsensoren einschränkt. Dies erklärt die Einschränkung durch die Zulassungsauflagen. Im Gegensatz zu Lidar- sind Radarsensoren weit weniger anfällig in Bezug auf Nebel (Hasirlioglu et al. 2017). Ein gleichzeitiger Einsatz von zwei Sensorprinzipien und die Fusion derer Signale liegt nahe und wird in diversen Veröffentlichungen empfohlen (z. B. Kutila et al. 2016).

Weitere Einflüsse durch die Umwelt werden an den Versuchen mit dem Laubbläser gezeigt. Da der Bus Objekte erkennt, aber keine Klassifizierung vornimmt, wird auf jedes Objekt gleich reagiert. Auch wenn von diesem keine Gefahr ausgeht. Dadurch wird der reguläre Betrieb des Busses behindert. Es ist anzunehmen, dass eine Objektklassifizierung die gezeigten Situationen erkennt und die Fahrt unbeeinflusst fortgesetzt würde.

Die Aussage eines Operators, dass neu aufgestellte Leitzylinder mit Reflektoren im Streckenabschnitt $\mathrm{C}$ die Fahrt beeinflussen, konnte nicht abschließend bewiesen oder entkräftet werden. Die Versuche mit seitlich stehenden Pylonen zeigen deutlich, dass die Reflektion von Gegenständen einen Einfluss auf die Abstandsmessung hat. Aufgrund der Ergebnisse wird ersichtlich, dass sich matte schwarze Oberflächen negativ auf die Erkennung von Gegenständen auswirken.

Die Versuchsergebnisse zeigen, dass die ausgewiesenen Funktionalitäten des Busses erfüllt werden. Allerdings decken diese nicht alle Verkehrssituationen ab, um ein unfallfreies Fahren zu gewährleisten. Daher ist der vorgeschriebene Einsatz eines Operators aus Sicherheitsgründen notwendig. Ebenfalls wird ersichtlich, dass eine erweiterte Sensorik sowohl bei der Sensortechnik als auch der Auswertesystematik große Teile der Sicherheitslücken des Busses Gen1 2.0 schließen kann. Zusätzlich würde dies unnötige Fahrtunterbrechungen verringern und den Fahrkomfort erhöhen, was den Einsatz im ÖPNV und die Akzeptanz des People Movers begünstigen würde.

\section{$5.4 \quad$ Fazit}

Vieles deutet darauf hin, dass der Bus sein Potenzial hinsichtlich autonomen Fahrens nicht ausschöpft bzw. nicht ausschöpfen darf. Die begrenzte Ausschöpfung wird zum Teil auch von den Zulassungsauflagen verursacht. Der Bus in diesem Entwicklungsstadium könnte aber auch nicht autonom fahren. Weder unfallfrei noch ohne Unterbrechungen. Somit 
kann der Shuttlebus keinesfalls als autonomes Fahrzeug eingestuft werden. Im Verbund mit den Zulassungsauflagen entspricht es einem Fahrzeug der Stufe L2 (vgl. Abschn. 5.2.1).

In Punkto Sicherheit haben die Zulassungsauflagen ihren Zweck erfüllt, da sie für einen unfallfreien Betrieb gesorgt haben, der ohne diese nicht gewährleistet wäre. Der einzige aufgetretene Unfall ist weder Eigenverschulden des Busses noch hätten die meisten ,echten" Fahrer diesen Unfall verhindert. Technisch wäre eine Vermeidung dieses Unfalls möglich, solange kein weiteres Fahrzeug hinter dem Bus steht.

Durch die Erprobung des Busses der ersten Generation Version 2.0 konnte gezeigt werden, dass es Verkehrssituationen gibt, in denen es ohne den vorgeschriebenen Operator zu einem Unfall gekommen wäre. Während der viermonatigen Erhebung wurde aber lediglich eine dieser Situationen dokumentiert (offener Kanaldeckel). Anhand dessen wird ersichtlich, dass die Wahrscheinlichkeit für solche Situationen sehr gering ist, der Operator dadurch aber für den unfallfreien Betrieb in Bad Birnbach unabdingbar ist.

Es ist davon auszugehen, dass durch den Einsatz der richtigen Umfeldsensoren, deren Positionierung und der entsprechenden Analysealgorithmen alle dokumentierten und in der Erprobung konstruierten Verkehrssituationen erkannt werden und ein Unfall verhindert wird. Eine Detektion von Objekten mit einer Umfelderfassung, die lediglich in einer Ebene erfolgt, macht einen unfallfreien Betrieb nicht möglich. Hierfür müssen Sensoren den gesamten Bereich vor dem Fahrzeug in Höhe und Breite erfassen und analysieren. Weiterhin darf die Bewertung des Umfeldes nicht zu einzelnen Zeitpunkten stattfinden, sondern muss die erfassten Signale in einen logischen Zusammenhang bringen. Ohne Objektverfolgung und Abschätzung für die nahe Zukunft würden Objekte, die sich in toten Winkeln der Sensoren befinden oder in diese hineinbewegen, missachtet und einen Unfall erzeugen.

Ein Einsatz der genannten Systeme wäre ebenfalls für einen kontinuierlichen Verkehrsfluss notwendig. Die Zulassungsauflagen schreiben die Bestätigung des Operators zum Losfahren an drei Stellen der Route vor, da die vorliegende Technik diese mit der notwendigen Sicherheit nicht autonom durchfahren kann. Weitere acht Bestätigungen muss der Operator beim Losfahren an den Haltestellen durchführen. In Bezug auf den Einsatz von autonomen Fahrzeugen im ÖPNV ist das ein Punkt, der in Zukunft gelöst werden muss. Ohne eigenständige Entscheidung durch den Bus, ob alle Fahrgäste ein- bzw. ausgestiegen sind und ein Schließen der Türen gefahrlos erfolgen kann, ist ein autonomer Einsatz im ÖPNV nicht möglich. Änderungen der umliegenden Infrastruktur können weitere Unterbrechungen des Verkehrsflusses bewirken, da der Bus die Orientierung verliert oder Objekte scheinbar den Weg versperren. In solchen Fällen muss der Operator die Fahrt manuell fortsetzen. Daran ist zu sehen, wie eng die Streckenauswahl und -vorbereitung mit der technischen Ausführung des Busses zusammenhängt.

Weiterhin ist ein Abweichen von der festgelegten Route entsprechend der Zulassungsauflagen nur durch den Operator zu bewerkstelligen, auch wenn die Technik des Busses dies ebenfalls könnte. In vielen der erhobenen Fälle wäre eine Weiterfahrt des Shuttlebusses ohne Operator nicht möglich. Dadurch würde der Shuttlebus selbst zum Verkehrshindernis und der Zweck der Personenbeförderung innerhalb festgelegter Zeiten nicht erfüllt. 
Im aktuellen Entwicklungsstadium des Busses und angesichts der damit einhergehenden Beschränkungen ist ein regulärer Betrieb im ÖPNV, der fahrplangebunden stattfindet, auch aus anderer Sicht nicht möglich. Wettereinflüsse schränken die Funktionen im Bereich der Orientierung und der Sicherheit derart ein, dass ein Betrieb bei Nebel, Regen und Schnee untersagt ist. Somit wird es zwangsläufig zu Ausfällen des automatisierten Betriebes kommen, deren Zeitpunkte des Auftretens und deren Dauer nicht vorhersagbar sind.

Nach heutigem Wissensstand sind Kombinationen verschiedener Sensortechniken und deren Datenfusion notwendig, um autonome sowie automatisierte Fahrfunktionen von Wetter unbeeinflusst zum Einsatz zu bringen.

Der Komfort für die Passagiere im Sinne einer gleichmäßigen Bewegung ist mit wenigen Ausnahmen sichergestellt. $3 \%$ aller dokumentierten Situationen wurden als Komfortsituation eingestuft. Da die Wahrnehmung allerdings rein subjektiven Aspekten unterliegt, kann durch die angewandte Datenerhebungsmethode keine belastbare Aussage getroffen werden. Zur Bewertung des Komforts müssen gezielte Befragungen der Fahrgäste durchgeführt und messbare Kriterien definiert werden (Kap. 6, Wintersberger et al.). Durch die Interviews mit den Operatoren und Gesprächen mit Fahrgästen sowie eigenen Erlebnissen bei der Mitfahrt wird allerdings deutlich, dass es Situationen gibt, die als störend, also unkomfortabel, empfunden werden.

Zum Anfahr- und Abbremsverhalten sind keine abwertenden Aussagen den Komfort betreffend bekannt, ebenso wenig zu Situationen, bei denen der Shuttlebus auf stehende Objekte reagieren muss. Das Annähern an stehende Verkehrsteilnehmer oder Objekte im Fahrschlauch sowie das Passieren dieser im Abstand des Perimeters d2 erfolgt mit gleichmäßigen Übergängen. Gegenüber herkömmlichen Bussen finden Geschwindigkeitsänderungen reproduzierbarer statt, worin ein deutlicher Vorteil des automatisierten Fahrzeuges liegt.

Eingeschränkt wird der Fahrkomfort bei Objekten, die sich in Abständen zum Shuttlebus bewegen, welche den Übergängen der einzelnen Sicherheitszonen entsprechen. Die vorhandene Schwankung an den Grenzen der Sicherheitszonen bewirkt einen ständigen Wechsel der Geschwindigkeit, welcher als störend empfunden werden kann.

Am Komfort zeigt sich, dass bei der Auslegung der Sensorsysteme Unterschiede zwischen stehenden und bewegten Objekten beachtet werden müssen. Die Interaktion mit bewegten Objekten erfordert deutlich komplexere Algorithmen als mit stehenden, um einen hohen Fahrkomfort zu realisieren. Weitere Forschungen hinsichtlich des Zusammenhangs von Fahrkomfort und technischer Auslegung des Gesamtsystems Shuttlebus sind hierfür notwendig.

Die technische Ausstattung des in Bad Birnbach eingesetzten Busses reicht nicht aus, um autonom fahrend einen unfallfreien Betrieb, den notwendigen Verkehrsfluss und einen durchgängig akzeptablen Komfort sicherzustellen. Zur Erfüllung dieser Punkte müssen die eingesetzte Sensortechnik und die Verarbeitung der erfassten Signale deutlich erweitert werden. Weiterhin müssen Funktionen freigegeben werden, die ein sicheres Verlassen der vorgegebenen Route ohne Operator ermöglichen. Zusätzlich muss die Ausfallsicherheit der Technik, die bei $22 \%$ der dokumentierten ungeplanten Unterbrechungen Ursache oder Folge war, deutlich erhöht werden. 
Die Zulassungsauflagen sind ausreichend, um selbstverschuldete Unfälle zu vermeiden, beeinflussen aber deutlich den Komfort der Fahrt und deren Verkehrsfluss. Bei der Erstellung der Zulassungsauflagen müssen Aspekte der Sicherheit und des Komforts gegeneinander abgewogen werden, wobei der Sicherheit oberste Priorität eingeräumt werden muss. Somit ist das Vorgehen der technischen Prüfstelle bei der Erstellung der Zulassungsempfehlung nachzuvollziehen. Es ist aber zu vermuten, dass es möglich ist, durch Lockerung der Auflagen den Komfort zu erhöhen, ohne die Unfallwahrscheinlichkeit zu vergrößern.

Durch das Projekt in Bad Birnbach und zukünftige Projekte müssen weitere Erfahrungen mit den technischen und rechtlichen Aspekten gesammelt werden, um autonomes Fahren unfallfrei, unterbrechungsfrei und komfortabel in großem Umfang anbieten zu können.

\section{Literatur}

4activesystems (2017) Advanced testing technologies for active safety systems to reduce road fatalities. 4activesystems, Traboch

Bijelic M, Gruber T, Ritter W (2018) A Benchmark for Lidar Sensors in Fog: Is Detection Breaking Down? IEEE. doi:https://doi.org/10.1109/IVS.2018.8500543

BfJ - Bundesamt für Justiz (2013) StVO § 22 Ladung Absatz 4

DB Regio (o. J.) Streckenverlauf. DB Regio Bus, Ingolstadt

Doric I (2017) A Generalised Approach to Active Pedestrian Safety Testing. Dissertation, University of Warwick

DSD (o. J.) Ultraflat Overrunable Robot for experimental ADAS Testing. Resource document. DSD homepage. http://www.dsd.at/index.php?option=com_content\&view=article\&id=259:ufo-en\&catid=16:crash-facilities\&lang=de. Zugegriffen: 20.10 .2019

EasyMile (o. J.) EasyMile EZ10 User Manual, EasyMile, Toulouse

Gängrich, P (2018) Autonome E-Busse für Hamburg - Das Projekt HEAT. Resource document. Hochbahn Blog. https://dialog.hochbahn.de/bus-in-zukunft/autonome-e-busse-fuer-hamburg-das-projekt-heat. Zugegriffen: 20.10.2019

Hasirlioglu S, Riener A (2017) Introduction to rain and fog attenuation on automotive surround sensors. IEEE. doi:https://doi.org/10.1109/ITSC.2017.8317823

Hasirlioglu S, Doric I, Kamann A, Riener A (2017) Reproducible Fog Simulation for Testing Automotive Surround Sensors. IEEE. doi:https://doi.org/10.1109/VTCSpring.2017.8108566

ioki (o. J.) Zulassungsprozess Bad Birnbach. Ioki, Frankfurt am Main

Krempl, S (2018) Berliner Charité und BVG testen autonome Kleinbusse. Resouce document. heise online. https://www.heise.de/newsticker/meldung/Berliner-Charite-und-BVG-testen-autonome-Kleinbusse-3789184.html. Zugegriffen: 20.10.2019

Kutila M, Pyykönen P, Ritter W, Sawade O, Schäufele B (2016) Automotive LIDAR sensor development scenarios for harsh weather conditions. IEEE. doi:https://doi.org/10.1109/ ITSC.2016.7795565

Neumann, P (2018) Charité - Darum müssen die autonomen BVG-Busse noch lernen. Resource document. Berliner Zeitung. https://www.berliner-zeitung.de/berlin/verkehr/charit\%C3\%A9-darum-muessen-die-autonomen-bvg-busse-noch-lernen-31799014. Zugegriffen: 20.10.2019 
Passauer Neue Presse (2018) Beim Ausparken mit autonomem Bus zusammengestoßen. Resource document. Passauer Neue Presse. https://www.pnp.de/lokales/landkreis_rottal_inn/pfarrkirchen/2955245_Beim-Ausparken-Zusammenstoss-mit-autonomem-Bus.html. Zugegriffen: 20.10.2019

SAE J3016. (2018). Taxonomy and definitions for terms related to driving automation systems for on-road motor vehicles, J3016_201806.

VDA - Verband der Automobilindustrie e. V. (2015) Automatisierung - Von Fahrerassistenzsystemen zum automatisierten Fahren.

VDI - Verein Deutscher Ingenieure e. V. (2018) Automatisiertes Fahren. VDI Statusreport, Düsseldorf

Open Access Dieses Kapitel wird unter der Creative Commons Namensnennung 4.0 International Lizenz (http://creativecommons.org/licenses/by/4.0/deed.de) veröffentlicht, welche die Nutzung, Vervielfältigung, Bearbeitung, Verbreitung und Wiedergabe in jeglichem Medium und Format erlaubt, sofern Sie den/die ursprünglichen Autor(en) und die Quelle ordnungsgemäß nennen, einen Link zur Creative Commons Lizenz beifügen und angeben, ob Änderungen vorgenommen wurden.

Die in diesem Kapitel enthaltenen Bilder und sonstiges Drittmaterial unterliegen ebenfalls der genannten Creative Commons Lizenz, sofern sich aus der Abbildungslegende nichts anderes ergibt. Sofern das betreffende Material nicht unter der genannten Creative Commons Lizenz steht und die betreffende Handlung nicht nach gesetzlichen Vorschriften erlaubt ist, ist für die oben aufgeführten Weiterverwendungen des Materials die Einwilligung des jeweiligen Rechteinhabers einzuholen.

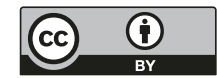

